\title{
A Novel Index Modulation Dimension based on Filter Domain: Filter Shapes Index Modulation
}

\author{
Majed Saad, Jacques Palicot, Faouzi Bader, Ali Chamas Al Ghouwayel and Hussein Hijazi
}

\begin{abstract}
A novel domain for Index Modulation (IM) named "Filter Domain" is proposed. This new domain generalizes many existing modulations and IM domains. In addition, a novel scheme "Filter Shape Index Modulation" (FSIM) is proposed. This FSIM scheme allows a higher Spectral Efficiency (SE) gain than the time and frequency IM dimensions in Single-Input Single-Output (SISO) systems. In the FSIM system, the bitstream is mapped using an Amplitude Phase Modulation (APM) as QAM or PSK, and an index of a filter-shape changing at the symbol rate. This filter $s$ hape, $b$ eing $c$ hanged a $t$ e ach symbol, enables a SE gain in SISO system without sacrificing a ny time or frequency resources. Compared to an equivalent $8 \mathrm{QAM}$ and 16QAM schemes and at the same SE, the FSIM with QPSK using 2 and 4 non-optimal filter $\mathbf{s}$ hapes a chieves a $\mathrm{g}$ ain of 3.8 $\mathbf{d B}$ and $1.7 \mathbf{d B}$ respectively at $\mathbf{B E R}=10^{-4}$, and this superiority is maintained in frequency selective fading channel compared to equivalent SISO-IM schemes. A low complexity detection scheme, approaching the maximum likelihood detector performance, is proposed along with a full performance characterization in terms of theoretical probability of filter i ndex e rror a nd B ER lower bound. Finally, FSIM can achieve better spectral and energy efficiencies when a filter bank and an IS I ca ncellation technique are optimally designed.
\end{abstract}

Index Terms-Index Modulation (IM), Filter Index Modulation, Filter Domain (FD), Filter Shape Index Modulation (FSIM), pulse-shaping filter, joint m aximum likelihood d etector, matched filter d etector, s pectral e fficiency, en ergy ef ficiency, SISO.

\section{INTRODUCTION}

Index Modulation (IM) has attracted tremendous attention in the last decade due to its potential enhancement in the system Spectral Efficiency ( SE) a nd/or E nergy E fficiency (E E). In addition, IM is well explored separately and jointly in the spatial, frequency, and temporal domains. The idea of IM is to convey additional information bits implicitly (Virtual Bits (VB)) in the index of the selected element among several possibilities. Note that by using an IM strategy, the receiver should be able to correctly detect the indexed element to deduce the associated VBs. For example, in the spatial IM domain, the indexed element is the antenna while it is the time slot and the frequency band/subcarrier in time IM domain and frequency IM domain, respectively.

M. Saad and J. Palicot are with Signal, Communication \& Embedded Electronics (SCEE) research group, CentraleSupélec \IETRCampus of Rennes, Cesson-Sévigné, 35510 Brittany-France e-mail: majed.saad@ieee.org, jacques.palicot@ centralesupelec.fr.

F. Bader is with Institut Supérieur d'Electronique de Paris, 75006 Paris, France email: faouzi.bader@isep.fr.

A. Chamas Al Ghouwayel is with the School of Engineering EFREI, 94800 Villejuif-Paris, France email: ali.ghouwayel@efrei.fr

H. Hijazi is with CCE department, Lebanese International University, Beirut, Lebanon email: hussein.hijazi@liu.edu.lb.
In the Multiple-Input Multiple-Output (MIMO) context, the different combinations of the indices of transmit or receive antennas are used to convey additional information bits by activating some transmit antennas or targeting some receive antennas. The Spatial Modulation (SM) activates a single antenna at the transmitter side to send an $M$-ary Amplitude Phase Modulation (APM) symbol [1]. Consequently, different versions of SM are proposed with a constant or variable number of active antennas to achieve a higher SE gain in [2], [3], and [4].

In the Single-Input Single-Output (SISO) context, the frequency IM domain conveys the VBs by the index of the frequency bands/subcarriers being selected when Single Carrier (SC)/Multi-Carrier (MC) waveforms are respectively used, e.g., OFDM with Sub-carrier IM (OFDM-SIM) [5]. In addition, the Time IM domain (TIM) counterpart was proposed with SC modulations motivated by their lower Peak to Average Power Ratio (PAPR) in SC-TIM [6]/SC- Frequency Division Multiple Access-IM (SC-FDMA-IM) [7] where the indexed element is the set of activated time slots/sub-carriers. However, most SISO-IM schemes suffer from low SE gain due to discarding some time/frequency resources.

Furthermore, the time and frequency IM domains are merged with the spatial domain to overcome their limited SE by benefiting from the multiplexing gain as in MIMO OFDMSIM [8]. A similar combination between these domains may also give a good tradeoff between multiplexing and diversity gains for better performance in a dispersive channel as in the Space-Time Shift Keying (STSK) [9]- [12], Space-Frequency Shift Keying (SFSK), and Space-Time-Frequency Shift Keying (STFSK) [13].

In brief, the spatial IM domain can enhance the SE/EE and reduce the cost of MIMO systems, depending on the spatial IM scheme being adopted, while the time/frequency IM domain strategies in SISO have a limited SE enhancement and, in some cases, a SE loss but with a better $\mathrm{EE}$ for low data rate applications and performance, especially in a dispersive channel.

In the context of the BRAVE project [14] [15], a higher $\mathrm{SE} / \mathrm{EE}$ is required to achieve a low power ultra-high wireless rates in sub-THz bands that enable many interesting scenarios [16]. In addition, the spatial IM domain considered in [17] [18] shows that using IM with power-efficient APM (e.g., Generalized SM-QPSK [19]) is more advantageous than the systems used in recent standards [20] where spatial multiplexing with high order QAM are adopted. Consequently, these results 
motivate us to propose a novel IM domain that enhances the $\mathrm{SE} / \mathrm{EE}$ in SISO prior to its MIMO exploitation. It is worth mentioning that each $\delta$ bits SE enhancement in SISO context will be multiplied by the multiplexing order in MIMO context.

In this paper, a novel domain for IM is proposed where a more significant SE enhancement is targeted in SISO with a better performance compared to the equivalent $M$-ary APM scheme without IM. Moreover, a novel scheme is proposed to achieve a higher SE enhancement compared to other IM domains in SISO. The main contributions of this paper are as follows:

1) A novel domain for index modulation, named filter domain (FD), is introduced where another degree of freedom for IM is presented. In addition, the derivatives of this FD and its different potential indexation techniques are discussed.

2) Within the filter domain/dimension, a novel modulation scheme named Filter Shape Index Modulation (FSIM) is proposed. To the best of our knowledge, this is the first time such an indexing dimension is used as a method for SE enhancement purpose. The pulse-shaping filter is indexed to convey additional information bits to those transmitted in the APM data symbols. The information bits in FSIM are mapped into both signal domain (any $M$-ary APM) and filter domain. We consider a filter bank with different shapes as a new approach rather than a single pulse shaping filter to further explore the indexation gain.

3) The FSIM system model is presented where a low complexity detector based on Matched Filter (MF) is proposed. Compared to the joint Maximum Likelihood (ML) detection that considers the joint detection of both filter shape index and APM symbols, the proposed detector performs firstly the filter shape detection and then the APM symbols being received. A prominent computational complexity reduction is obtained.

4) An Inter-Symbol Interference (ISI) cancellation is proposed to eliminate the effect of the ISI introduced by the designed filter shapes.

5) Theoretical analysis and Monte Carlo simulations substantiating the efficiency of the proposed FSIM scheme is presented. A lower bound for the error probability of the filter index is derived. In addition, the APM error is incorporated to truly characterize the FSIM system performance in terms of total Symbol-Error Rate (SER) and Bit-Error Rate (BER). This lower bound can be evaluated numerically with any number of filters and $M$-ary APM scheme. Simulation results demonstrate the superior performance of FSIM compared to the other systems of the same SE with/without IM in AWGN and frequency selective fading channels.

The rest of the paper is organized as follows. Section II introduces the novel Filter domain for IM that generalizes most of SISO-IM schemes. The novel FSIM transceiver and the filter bank design requirements are presented in section III, where different detectors are used, and an ISI cancellation technique is proposed. In Section IV, theoretical performance analysis and derivation of the analytical lower bound are given to validate the proposed scheme. A comparison, based on numerical and analytical results, with the widely used existing equivalent schemes, is presented in Section V. Finally, Section VI concludes the paper.

The notations are as follows: Bold lower case is used for vectors. The characters $*$ and $\star$ denotes the convolution and cross correlation respectively. $f^{*}$ denotes the complex conjugate of $f .\langle x, y\rangle$ stands for the dot-product. $\mathcal{C N}\left(\mu, \sigma^{2}\right)$ denotes the complex normal distribution of a random variable having mean $\mu$ and variance $\sigma^{2} .\lfloor x\rfloor(\lceil x\rceil)$ denotes the floor (ceil) function that means the largest (smallest) integer less (greater) than or equal to $x$. $\|$.$\| stands for the Frobenius norm.$ $\left(\begin{array}{l}n \\ k\end{array}\right)$ denotes the binomial coefficient. The probability of an event is denoted by $P($.$) and the Probability Density Function$ (PDF) is denoted by $p($.$) . \mathbb{E}[$.$] denotes the expectation.$

\section{Filter Domain FOR Index MOdulation}

In this section, the SE of the different existing SISO schemes is reviewed, since our proposed IM domain and FSIM scheme aim mainly to enhance the SE in SISO mode and generalize the time/frequency IM domains.

In general, the time and frequency IM domains have limitations in the SE enhancement for high data rate applications. This limitation is due to the discarding of some available time and frequency resources. The time domain-IM, which activates a time slot to convey information bits, appeared from many decades in several schemes as On-Off Keying (OOK), Pulse Position Modulation (PPM) without APM [21] and Generalized PPM (GPPM) with APM [22]. Then, the SE is enhanced by activating a fixed number of time slots in SCIM [6], which is proposed with frequency domain equalization (FDE) inspired by the frequency domain counterpart OFDMSIM [5] [26]. Similarly, the Generalized FDM-SIM (GFDMSIM) [27] uses the index of activated frequency bands for the same purpose. Their SE without pulse-shaping rolloff factor effect is expressed in Table I, where the $N$ time slots or subcarriers/frequency bands without the cyclic prefix elements of length $N_{C P}$ is divided into $G$ groups of $N_{g}$ elements $\left(N=N_{g} G\right)$, and a fixed $N_{a}\left(N_{a} \leq N_{g}\right)$ is activated to convey information in IM. The indexation is performed using an acceptable group size $N_{g}$ to avoid the high detection complexity with a large $N$. Inspired by OFDM-SIM, the multicarrier differential chaos shift keying (DCSK) modulation is combined with IM by conveying bits using the activated carrier index and transmitting zero symbols on non-activated ones [30]. Similarly, the IM approach is used with spreading spectrum techniques by selecting one of the predefined spreading codes in Generalized Code Index Modulation [31]. Other IM idea is also investigated in OFDM with Sub-carrier Number Modulation (OFDM-SNM) [32] [33], where only the number of activated sub-carriers ( $N_{a}$ value) conveys the VBs. The allowed $N_{a}$ values for a certain OFDM-SNM system is represented in the set $\phi_{g}$, which can contain all possible values in the extreme case $\left(\phi_{g}=\left\{1,2, \ldots, N_{g}\right\}\right.$ where its SE remains lower than conventional $M$-ary APM). 
TABLE I: SE of several existing SISO schemes

\begin{tabular}{|c|c|c|c|c|}
\hline IM Domain & $\mathrm{SC} / \mathrm{MC}$ & System Name & Spectral Efficiency & $\begin{array}{l}\text { Inherited } \\
\text { Interference }\end{array}$ \\
\hline- & SC & Linear APM:QAM, PSK,... & $\log _{2} M$ & No \\
\hline- & $\mathrm{SC} / \mathrm{MC}$ & SC-FDE/OFDM & $\frac{N \log _{2} M}{N+N_{C P}}$ & No \\
\hline Time & $\mathrm{SC}$ & OOK & 1 & No \\
\hline Time & $\mathrm{SC}$ & PPM [21] & $\frac{\left\lfloor\log _{2} N_{g}\right\rfloor}{N_{g}}$ & No \\
\hline Time & $\mathrm{SC}$ & GPPM [22] & $\frac{\left\lfloor\log _{2} N_{g}\right\rfloor+\log _{2} M}{N_{g}}$ & No \\
\hline Time & $\mathrm{SC}$ & NOMA: IMMA [23] & $\frac{N_{u}\left(\left\lfloor\log _{2} N_{g}\right\rfloor+\log _{2} M\right)}{N_{g}}$ & YES \\
\hline Time & $\mathrm{SC}$ & NOMA: QIMMA [24] & $\frac{N_{u}\left(2\left\lfloor\log _{2} N_{g}\right\rfloor+\log _{2} M\right)}{N_{g}}$ & YES \\
\hline Frequency & $\mathrm{SC}$ & NOMA: IM-OFDMA [25] & $\frac{N_{u}\left(\log _{2} M+\left\lfloor\log _{2}\left(\begin{array}{l}N_{g} \\
N_{a}\end{array}\right)\right\rfloor\right)}{N_{g}}$ & YES \\
\hline $\begin{array}{c}\text { Time } \\
\text { Frequency }\end{array}$ & $\begin{array}{l}\mathrm{SC} \\
\mathrm{MC}\end{array}$ & $\begin{array}{l}\text { FDE SC-TIM [6], SC-FDMA-IM [7] } \\
\text { OFDM-SIM [5] [26], GFDM-SIM [27] }\end{array}$ & $\frac{G\left(N_{a} \log _{2} M+\left\lfloor\log _{2}\left(\begin{array}{l}N_{g} \\
N_{a}\end{array}\right)\right\rfloor\right)}{N+N_{C P}}$ & No \\
\hline Frequency & MC & OFDM-VSIM [28] & $\begin{array}{l}\frac{\left.G\left(\left\lfloor\log _{2}\left(\sum_{N_{a} \in \phi_{g}} M^{N_{a}}\left(\begin{array}{c}N_{g} \\
N_{a}\end{array}\right)\right)\right)\right\rfloor\right)}{N+N_{C P}} \\
\text { Extreme case: } \frac{G\left\lfloor\log _{2}(M+1)^{N_{g}}\right\rfloor}{N+N_{C P}}\end{array}$ & No \\
\hline Frequency & MC & OFDM-QSIM [28] & $\frac{G\left(N_{a} \log _{2} M+2\left\lfloor\log _{2}\left(\begin{array}{l}N g \\
N_{a}\end{array}\right)\right\rfloor\right)}{N+N_{C P}}$ & No \\
\hline Frequency & MC & OFDM-HIQ-SIM [29] & $\frac{G\left(\left(N_{a_{I}}+N_{a_{Q}}\right) \log _{2} \sqrt{M}+\left\lfloor\log _{2}\left(\begin{array}{c}N_{g} \\
N_{a_{I}}\end{array}\right)\left(\begin{array}{c}N_{g} \\
N_{a_{Q}}\end{array}\right)\right\rfloor\right)}{N+N_{C} P}$ & No \\
\hline Frequency & MC & OFDM-SNM [32] [33] & $\begin{array}{c}\frac{G\left(N_{a} \log _{2} M+\left\lfloor\log _{2} N_{g}\right\rfloor\right)}{N+N N_{C P}}, 1 \leq N_{a} \leq N_{g} \\
\text { Average: } \frac{G\left(0.5\left(1+N_{g}\right) \log _{2} M+\left\lfloor\log _{2} N_{g}\right\rfloor\right)}{N+N_{C P}}\end{array}$ & No \\
\hline $\begin{array}{c}\text { Time } \\
\text { Frequency }\end{array}$ & $\begin{array}{l}\mathrm{SC} \\
\mathrm{SC}\end{array}$ & $\begin{array}{l}\text { FTN-IM [34] } \\
\text { SeFDM-IM [35] }\end{array}$ & $\frac{1}{\nu} \frac{G\left(N_{a} \log _{2} M+\left\lfloor\log _{2}\left(\begin{array}{c}N_{g} \\
N_{a}\end{array}\right)\right\rfloor\right)}{N+N_{C P}}$ & YES \\
\hline Modulation & $\mathrm{SC}$ & DM-FTN-IM [36] & $\frac{1}{\nu} \frac{G\left(N_{a} \log _{2} M_{A}+\left(N_{g}-N_{a}\right) \log _{2} M_{B}+\left\lfloor\log _{2}\left(\begin{array}{l}N_{g} \\
N_{a}\end{array}\right)\right\rfloor\right)}{N+N_{C P}}$ & YES \\
\hline $\begin{array}{l}\text { Modulation } \\
\text { Modulation }\end{array}$ & $\begin{array}{l}\mathrm{SC} \\
\mathrm{MC}\end{array}$ & $\begin{array}{c}\text { DM-SC-IM [37] } \\
\text { DM-OFDM-SIM [38] }\end{array}$ & $\frac{G\left(N_{a} \log _{2} M_{A}+\left(N_{g}-N_{a}\right) \log _{2} M_{B}+\left\lfloor\log _{2}\left(\begin{array}{l}N_{g} \\
N_{a}\end{array}\right)\right\rfloor\right)}{N+N_{C P}}$ & No \\
\hline Modulation & MC & DM-OFDM-VSIM [39] & $\frac{\left.G\left(\left\lfloor\log _{2}\left(\sum_{N_{a} \in \phi_{g}} M_{A}^{N_{a}} M_{B}^{N_{g}-N_{a}}\left(\begin{array}{l}N_{g} \\
N_{a}\end{array}\right)\right)\right)\right\rfloor\right)}{N+N_{C P}}$ & No \\
\hline Modulation & MC & MM-OFDM-IM [40] & $\frac{G\left(\left\lfloor\log _{2} N_{g} !\right\rfloor+N_{g} \log _{2} M\right)}{N+N_{C} P}$ & No \\
\hline Modulation & $\mathrm{MC}$ & MM-OFDM-QIM [40] & $\frac{G\left(2\left\lfloor\log _{2} N_{g} !+N N_{g} \log _{2} M\right)\right.}{N+N_{C P}}$ & No \\
\hline Modulation & MC & GMM-OFDM-IM [41] & $\begin{array}{c}\frac{G\left(\left\lfloor\log _{2} N_{g} !\right\rfloor+\sum_{k=1}^{K} n_{k} \log _{2} M_{k}\right)}{N+N_{C P}} \text { where } \\
\sum_{k=1}^{K} n_{k}=N_{g} \text { and } M_{1}>M_{2}>\ldots M_{K}\end{array}$ & No \\
\hline Frequency & MC & $\mathcal{L}$-OFDM-SIM [42] & $\frac{\left.G \mathcal{L}\left(\left\lfloor\log _{2}\left(\begin{array}{c}N_{g}-N_{a}(\mathcal{L}-1) \\
N a\end{array}\right)\right\rfloor+N_{a} \log _{2} M\right)\right)}{N+N_{C P}}$ & No \\
\hline
\end{tabular}

Moreover, the time and frequency IM domain are adopted with non-orthogonal resources in Faster-Than-Nyquist (FTNIM) (time domain-IM) and Spectral efficient FDM (SeFDM) (frequency domain-IM), respectively. These systems suffer from inherited interference, but it can compensate some of the SE loss or limited gain of their orthogonal version, depending on the compression factor $\nu$ at the price of performance degradation.

Similarly, the IM Multiple access (IMMA) [23] and IM Orthogonal Frequency Division Multiple Access (IM-OFDMA) [25] exploit the appealing advantage of IM in proposing a new Non-Orthogonal Multiple Access (NOMA) in time and frequency, respectively. Note that IMMA is similar to GPPM, whereas it allows resource sharing among $N_{u}$ users. How- ever, the frequency domain counterpart IM-OFDMA allows to activate $N_{a}$ subcarriers in contrast to IMMA that uses a single time slot. The SE enhancement of IMMA is doubled in Quadrature IMMA (QIMMA) [24] by performing separate indexation on the In-phase (I) and Quadrature (Q) components. In other words, QIMMA transmits the real and imaginary parts of the complex symbol through different resources unless the VBs for I and Q are the same. Consequently, these nonorthogonal schemes with a convenient configuration achieve a performance gain that can vanish due to collisions when many users transmit during the same time/frequency resources. Thus, they provide a trade-off between the SE system enhancement and the collision probability that are affected by the maximum number of users in each time/frequency chunk. 
Furthermore, several SE enhancements for these SISO-IM are enabled by allowing the activation of a variable number of sub-carriers in OFDM-Variable SIM (OFDM-VSIM) [28] and/or independent sub-carriers activation with the same $N_{a}$ between the I and Q components in OFDM-Quadrature SIM (OFDM-QSIM) [28] or different $N_{a}$ between them in OFDMHybrid IQ-SIM (OFDM-HIQ-IM) [29]. For the same reason, the Layered-OFDM-SIM ( $\mathcal{L}$-OFDM-SIM) is proposed in [42] while taking advantage of modulation type IM domain that uses distinguishable modulations between different layers. In each group of $N_{g}$ subcarriers, the $\mathcal{L}$-OFDM-SIM scheme activates $N_{a}$ subcarriers per layer of $N_{g}-N_{a}(\mathcal{L}-1)$ elements. This $\mathcal{L}$-OFDM-SIM activates more subcarriers in total $\left(G \mathcal{L} N_{a}\right)$ compared to OFDM-SIM $\left(G N_{a}\right)$, and the former performs another SIM on the remaining non-activated subcarriers successively on the subsequent $\mathcal{L}-1$ layers within each group that help to enhance the SE of OFDM-SIM.

However, the previous techniques do not use all available time/frequency resources that limit the SE enhancement. Hence, a dual-mode (DM) IM is introduced to better compensate for the SE loss or limited gain. Indeed, the non-activated slots/bands are exploited to send different and distinguishable APM symbols by means of modulation type IM, so $M_{A}$ and $M_{B}$-ary APM schemes are used respectively during the primary and secondary activated time slots in DM-SC-IM [37] and DM-FTN-IM [36], and frequency sub-carriers in DMOFDM-IM as discussed in [38] [39]. These DM-IM techniques convey information by the used modulation type, and this idea is extended to Multi-Mode (MM) OFDM-IM (MM-OFDMIM), where each subcarrier within a group of $N_{g}$ elements transmits a symbol from different constellation sets, and the permutation of these modulation conveys the VBs, similarly for MM-OFDM-Quadrature IM (MM-OFDM-QIM) but with different indexation on I and Q. Afterward, a Generalized MM-OFDM-IM is proposed in [41] to provide a more flexible choice for SE compared to MM-OFDM-IM, by adjusting the number of subcarriers $n_{k}$ that use the same modulation order $M_{k}$ (the special case MM-OFDM-IM is obtained when all $M_{k}$ are equal to $M$ as shown in Table I). For more details, the reader is referred to the survey [43] that summarizes most of these IM schemes and those in the spatial IM domain.

It is clear from Table I that the SISO-IM schemes that do not fully use the time and frequency resources suffer from $\mathrm{SE}$ reduction in most configurations, while the dual and multimode versions that use all resources can achieve limited SE enhancement. Although the SE for single-mode IM schemes is limited, they are promising for low data rate applications, such as the Low Power Wide Area Internet of Things (IoT) devices and machine-to-machine communications, since a better performance can be achieved with some of them compared to conventional APM schemes. In addition, a theoretical EE can be enhanced with low data rate applications to reach $50 \%$ for the same SE with $M=2 \mathrm{BPSK}, N_{g}=4$, $N_{a}=2$ as presented in SC-FDMA-IM [7]. However, their $\mathrm{EE}$ is dramatically reduced compared to conventional APM schemes (QAM) for high data rate applications. This lower $\mathrm{EE}$ is due to the need for higher power (SNR) to maintain the same SE, by using either higher $M$-ary APM when not all resources are used according to Table I, or non-orthogonal schemes with inherited interference.

Aiming at further enhancing the SE gain, the FD is proposed in this section. It will be first applied to SISO systems prior to its exploitation in MIMO context. It is worth mentioning that FD can include all kinds of communication systems because all of these systems have at least one filter used for band-limitation of the signal. In addition, the FD-IM domain allows the indexation of several filter properties to convey information bits with or without an APM symbol. Adopting the FD indexation strategy, one can cover as many schemes as it can be imagined to convey VBs by indexing: the bandwidth, the roll-off factor, the filter shapes, the filter time shift,...etc.

Hence, the frequency IM domain can be seen as a special case of the FD because the activation/deactivation of a frequency band or a subcarrier is just an application of a bandpass/zero filters in single-mode IM. Similarly, the time IM domain counterpart can also be considered as a special case of the FD, where the transmit filter is shifted by multiple symbol periods without allowing overlapping of different symbols. Note that in the single-mode time IM domain, the transmitter sends APM symbols only during activated time slots where a non-zero filter is used. In DM-IM, the transmission of data symbols in all time slots or subcarriers is enabled by using distinguishable APM schemes between primary and secondary activated resources. The DM-IM systems in [36]- [38] consider two distinguishable constellation alphabets drawn from the inner and outer constellation points of different average power levels using the same or different normalized constellation points. Note that DM-IM can be achieved by using the same normalized APM alphabets followed by different filters to change power and phase, by using different APM constellations with the same filter or using both different APM constellations and filters between primary and secondary active resources. The filter IM domain generalizes the single-mode IM by selecting only different filters between active and nonactive sub-carriers or time slots. However, the filter IM domain also needs to select different APM mapper (constellation alphabets) between the different active resources to generalize all DM-IM and MM-IM cases. Therefore, the filter IM domain can generalize most of SISO IM schemes.

Furthermore, FD-IM also includes the conventional modulation schemes as the Pulse-Position Modulation (PPM), PulseWidth Modulation (PWM), On-Off Keying (OOK) because they convey bits by means of indexing a filter property. Hence, FD-IM generalizes most of SISO-IM schemes, and its exploration allows more innovation in communication systems.

In this paper, many possible indexations in FD (e.g., the filter roll-off) are not explored, currently, because they involve a bandwidth variation, and/or they cannot be modified and detected at the symbol rate, and thus negatively impacting the SE, which is contradictory with our ultimate goal: the SE enhancement. In the following, we will introduce our proposed novel scheme in this IM domain to convey additional information by indexing the filter shapes. This scheme uses all frequency and time resources where the filter shape can be changed at each symbol period to maximize the possible SE enhancement. 


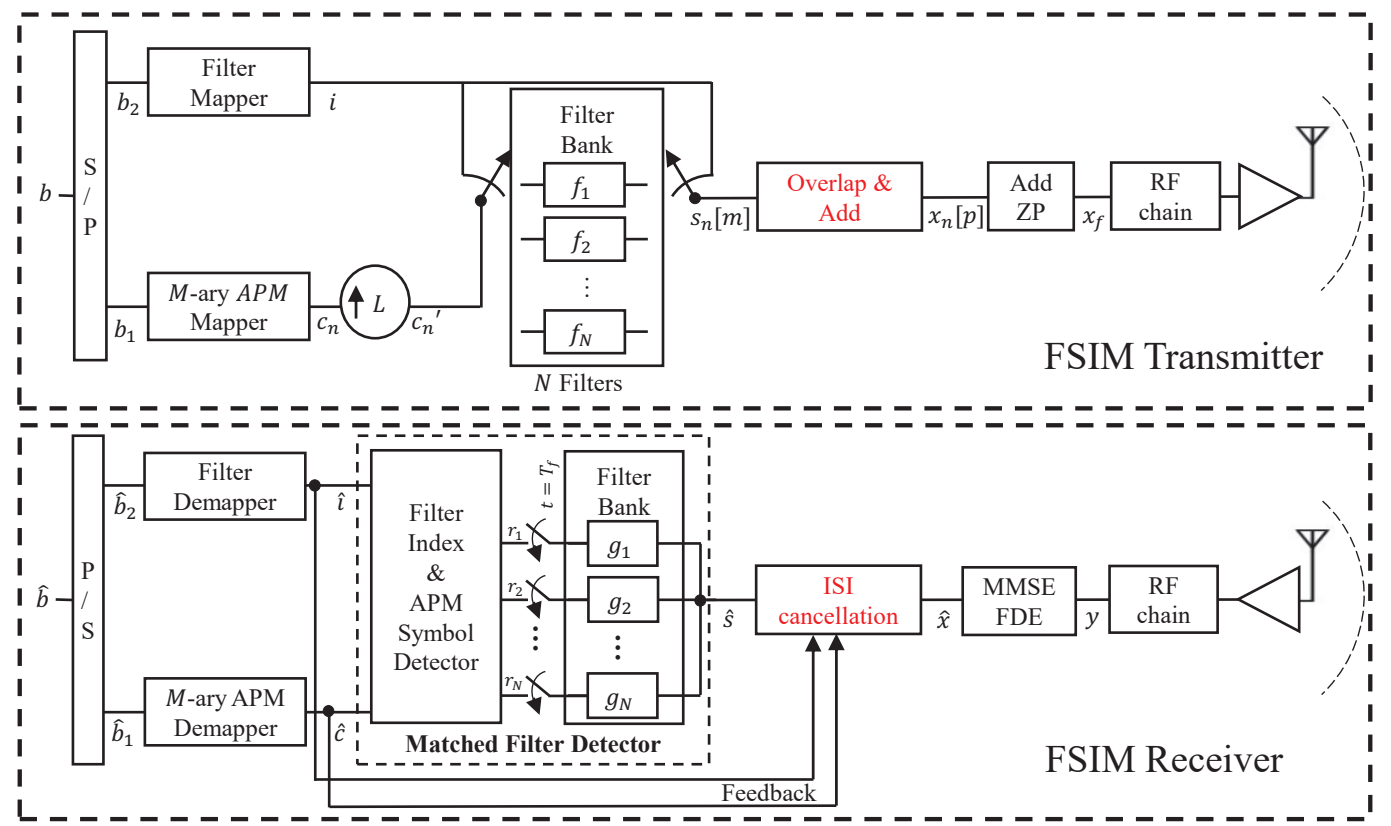

Fig. 1: System model of the FSIM-based transmitter and receiver with MF-based detector using $N$ filters of length $L$ and $M$-ary APM. Note that the detector can be replaced by the joint ML detector.

\section{Filter Shape Index Modulation}

\section{A. FSIM Transmitter}

The proposed system considers a SISO transmission mode, where the binary source information sequence $b$ is divided into two streams $b_{1}$ and $b_{2}$ as shown in Fig. 1 . The bit-stream $b_{1}$ is mapped by the $M$-ary APM, such as QAM or PSK, etc. The bit-stream $b_{2}$ is encapsulated in the index $i$ of the selected filter. Then, the selected filter $\mathbf{f}_{i}[m]$ of length $L$ samples is used as a pulse-shaping filter where $m$ is the sample index taking values between 0 and $L-1$. The filter is truncated in the time domain to $\eta$ APM symbols and sampled with a rate of $\lambda$ samples per symbol, which yields $L=\eta \cdot \lambda+1$ samples. $L$ is restricted to be odd in order to have a linear phase filter without a half sample period shift.

We suppose that there is a filter bank with $N$ distinguishable filters as shown in Fig. 1 ( $N$ is a power of 2). Accordingly, the number of bits per FSIM symbol $\mathcal{L}_{\text {FSIM }}$ can be expressed as:

$$
\mathcal{L}_{\text {FSIM }}=\log _{2} N+\log _{2} M .
$$

Thus, the SE is enhanced by $\log _{2} N$ compared to conventional $M$-ary APM systems thanks to the filter indexation at each symbol period.

The output of the filter bank for the $n$-th APM complex symbol $c_{n}$ is denoted by signals $s_{n}[m]$ and given as follows:

$$
s_{n}[m]=\left(f_{i_{n}} * c_{n}^{\prime}\right)[m]=c_{n} f_{i_{n}}[m]
$$

where $c_{n}^{\prime}$ is an up-sampled version of $c_{n}$ by a factor $L$, and $i_{n} \in\{0,1, \ldots, N-1\}$ is the index of the filter being selected for the $n^{\text {th }}$ symbol according to the bit-stream $b_{2}$.

Then, the signals $s_{n}[\mathrm{~m}]$ are passed through the OverlapAdd (OLA) block as shown in Fig. 1 in order to generate the $\lambda$ samples for each APM symbol. The $\lambda$ desired samples for the $n^{\text {th }}$ APM symbol $c_{n}$ is given by $x_{n}[p]$ as follows:

$$
x_{n}[p]=\sum_{n^{\prime}=n-\lceil\eta / 2\rceil}^{n+\lfloor\eta / 2\rfloor} s_{n^{\prime}}\left[p-\left(n^{\prime}-n\right) \lambda\right],
$$

where the index $p=p_{\text {center }}-\lceil\lambda / 2\rceil+1, \ldots, p_{\text {center }}+\lfloor\lambda / 2\rfloor$ and the index of the middle desired sample is $p_{\text {center }}=\frac{L-1}{2}$.

An example for the OLA with $\eta=6$ is illustrated in Fig. 2. Note that each row in this figure represents the $L$ output samples of the filter bank for the $n^{t h}$ symbol $c_{n}$ which contains: the desired $\lambda$ samples $\mathbf{s}_{n}$ at the center and the undesired samples $\mathbf{I}_{j}^{n}$ for $j= \pm 1, \pm 2, \pm 3$. The $\lambda$ samples $\mathbf{s}_{n}$ will be affected and interfered by the undesired samples of the $\lceil\eta / 2\rceil=3$ APM symbols prior the instant $n$ (for $j>0$ ) and of $\lfloor\eta / 2\rfloor=3$ APM symbols posterior to the instant $n$ (for $j<0$ ) as depicted in the red vertical box (for $n=3$ ) of Fig. 2. Hence, the equation (3) can be rewritten in terms of interference as:

$$
\mathbf{x}_{n}[p]=\mathbf{I}_{\lceil\eta / 2\rceil}^{n-\lceil\eta / 2\rceil}+\ldots+\mathbf{I}_{1}^{n-1}+\mathbf{s}_{n}+\mathbf{I}_{-1}^{n+1}+\ldots+\mathbf{I}_{-\lfloor\eta / 2\rfloor}^{n+\lfloor\eta / 2\rfloor}
$$

Note that the conventional transceiver with any $M$-ary APM schemes can be considered as a special case from our proposed system where all the filters in the bank are all substituted by the same Nyquist pulse-shaping filter e.g., RootRaised Cosine (RRC). In other words, the proposed system is equivalent to conventional transceiver when using the same Nyquist filter for all symbols, and this proof is presented in Appendix A. Finally, a frame $\mathbf{x}_{f}$ is created by padding the signal of $N_{s}$ FSIM symbols by a zero-prefix of length $\lambda N_{Z P}$ samples in order to eliminate the inter-frame interference: $\mathbf{x}_{f}=\left[0_{1}, \ldots, 0_{N_{Z P}}, \mathbf{x}_{-\lfloor\eta / 2\rfloor}, \ldots, \mathbf{x}_{\left\lceil N_{s}-1+\eta / 2\right\rceil}\right]$. Hence, the SE of FSIM is attenuated by a factor of $N_{s} /\left(N_{s}+N_{Z P}\right)$.

\section{B. FSIM Receiver}

The receiver scheme proposed by our approach is represented in Fig. 1, where the received signal $y$ is expressed in 


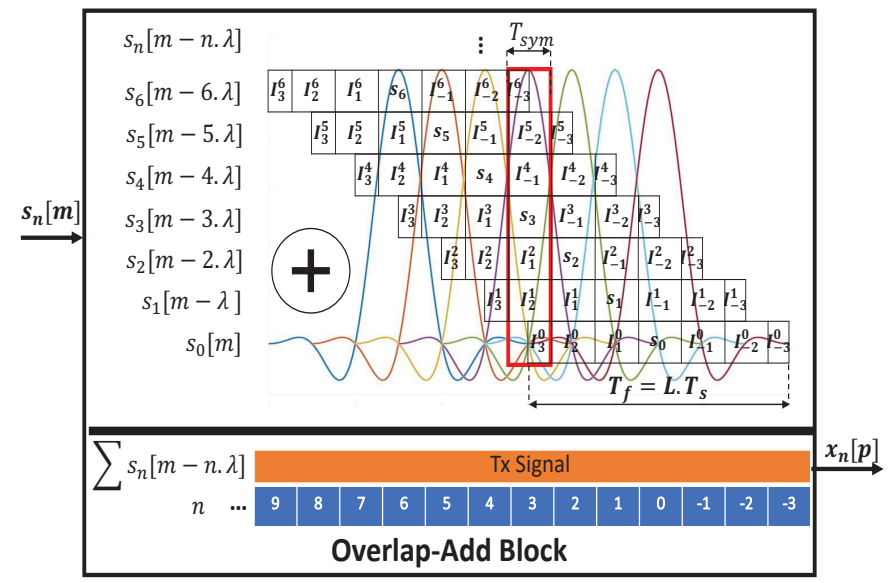

Fig. 2: An example for the Overlap-Add block in the proposed scheme assuming $\eta=6$ symbols.

time domain as:

$$
y(t)=\left(h * x_{f}\right)(t)+v(t),
$$

where $h(t)$ is the impulse response of a multipath frequencyselective fading channel with $J$ paths and maximum delay spread of $(J-1) T_{\text {sym }}$, where $T_{\text {sym }}=\lambda \cdot T_{s}$ is the APM symbol period and $T_{s}$ is the sampling period. Note that $N_{Z P} \geq(J-$ 1) in order to avoid the inter-frame interference. $v(t)$ is the Additive White Gaussian Noise (AWGN) with zero mean and variance $\sigma_{v}^{2}$, i.e, $\mathcal{C N}\left(0, \sigma_{v}^{2}\right)$. The power of transmitted symbols $c_{n}$ and the used filters $f_{i_{n}}$ are normalized: $\mathbb{E}\left[\left\|c_{n}\right\|\right]=1$ and $\left\|\mathbf{f}_{i}\right\|^{2}=\sum_{m=0}^{L-1} f_{i}^{2}[m]=1$, respectively.

Firstly, the receiver compensates for the fading channel distortion on all received samples that contain the implicit information of the selected filter index. For each received frame, the zero-prefix is removed, then a linear equalizer like Minimum Mean-Square Error-FDE (MMSE-FDE) [44] is used to mitigate the channel effect and recover the signal $\hat{x}$ as depicted in Fig. 1.

Afterward, the equalized signal $\hat{x}$ is passed to the ISI estimation and cancellation that remove the controlled ISI from the used filter shapes. The ISI cancellation is required if the used filters don't satisfy the Nyquist ISI criterion. The condition for zero ISI in AWGN channel is:

$$
\left(f_{i} * g_{i}\right)\left(n . T_{\text {sym }}\right)= \begin{cases}1 & n=0 \\ 0 & n \neq 0\end{cases}
$$

for any integer $n$ and $i=1, \ldots, N$, where $g_{i}$ is the impulse response of the MF. Note that when this condition is satisfied, only one pulse has an effect at the integer multiple of the symbol period, as shown in Fig. 2. However, this condition is relaxed in the proposed system when the target is to enhance the SE of $M$-ary APM, and then ISI will occur naturally. Therefore, an ISI cancellation process is mandatory before detecting the APM symbol and the selected filter index. The APM symbol and index detection can be performed jointly using the joint ML detector or separately using the MF detector. The proposed detectors are described in the following.

1) Joint ML Detector: The equalized signal $\hat{x}$ is fed to the ISI cancellation block that aims at regenerating free-ISI signals $\hat{\mathbf{s}}$ composed each of $L$ samples. These signals $\hat{\mathbf{s}}$ are then fed to the detector to jointly recover the filter index and the transmitted symbols. The detection can be performed using an ML detector that makes an exhaustive search over all possible APM symbols and filters $\mathbf{f}_{i}$ combinations as described below:

$$
\{\hat{\imath}, \hat{c}\}=\arg \min _{\mathbf{f}_{i} \in \psi, c \in \chi}\left\|\hat{\mathbf{s}}-\mathbf{f}_{i} * c\right\|^{2}
$$

where $\psi$ and $\chi$ denote the set of $N$ filter's shapes and the $M$ ary APM constellation, respectively. $\hat{\imath}$ and $\hat{c}$ are the estimated filter index of $\mathbf{f}_{i}$ and the estimated APM symbol, respectively. Such a receiver's system model is similar to that illustrated in Fig. 1 but by replacing the MF-based detector by an ML detector.

2) Matched Filter-based Detector: The detection of the selected filter index can be performed using a bank of MFs or a bank of correlators. This paper will consider the MFbased detector, knowing that both detectors provide the same results [46]. Figure 1 depicts the system model where the detection is performed after the ISI cancellation. The detector is composed of $N$ matched filter $g_{k}$ where $g_{k}(t)=f_{k}\left(T_{f}-t\right)$ with $0 \leq t \leq T_{f}, T_{f}=L . T_{s}$. The outputs of these filters $r_{k}(t)$ are given by:

$$
\begin{aligned}
r_{k}(t) & =\int_{0}^{t} \hat{s}(\tau) g_{k}(t-\tau) d \tau, \quad k=1,2, \ldots, N \\
& =\int_{0}^{t} \hat{s}(\tau) f_{k}\left(T_{f}-t+\tau\right) d \tau .
\end{aligned}
$$

These filter outputs are evaluated at the instant $T_{f}$

$$
r_{k}=\int_{0}^{T_{f}} \hat{s}(\tau) f_{k}(\tau) d \tau, \quad k=1,2, \ldots, N .
$$

Thus, the decision on the selected filter index, being used at the transmitter, is based on selecting the largest energy of the sampled MF outputs as follows:

$$
\hat{\imath}=\arg \max _{k}\left\|r_{k}\right\|^{2} .
$$

Once the filter index is detected, $r_{\hat{\imath}}$ is decoded by using the APM detector that determines the ML transmitted symbol $\hat{c}$ in the constellation set $\chi$. Note that any other APM detector can be used. Finally, the detected APM symbol $\hat{c}$ and the filter index $\hat{\imath}$ are sent to the corresponding demappers to deduce the bit-stream $\hat{b}_{1}$ and $\hat{b}_{2}$, respectively.

3) ISI Estimation and Cancellation: The principle of FSIM is to convey additional information bits through the index of the transmission filter being selected at the transmitter. Thus, this modulation's success is based on the capability of detecting correctly at the first stage, the index of the selected filter $f_{i}$. For this reason, the filter bank cannot contain only Nyquist filters due to the inherent high cross-correlation between the possible Nyquist filters. One of the solutions that could allow the detection of correlated Nyquist filter is to use the same filter shape for many successive symbols. However, this solution contradicts the FSIM approach, aiming to increase the SE in contrast to other SISO-IM schemes. Therefore, the filter bank used in this modulation is expected to generate ISI but predictable depending on the filter shapes. 


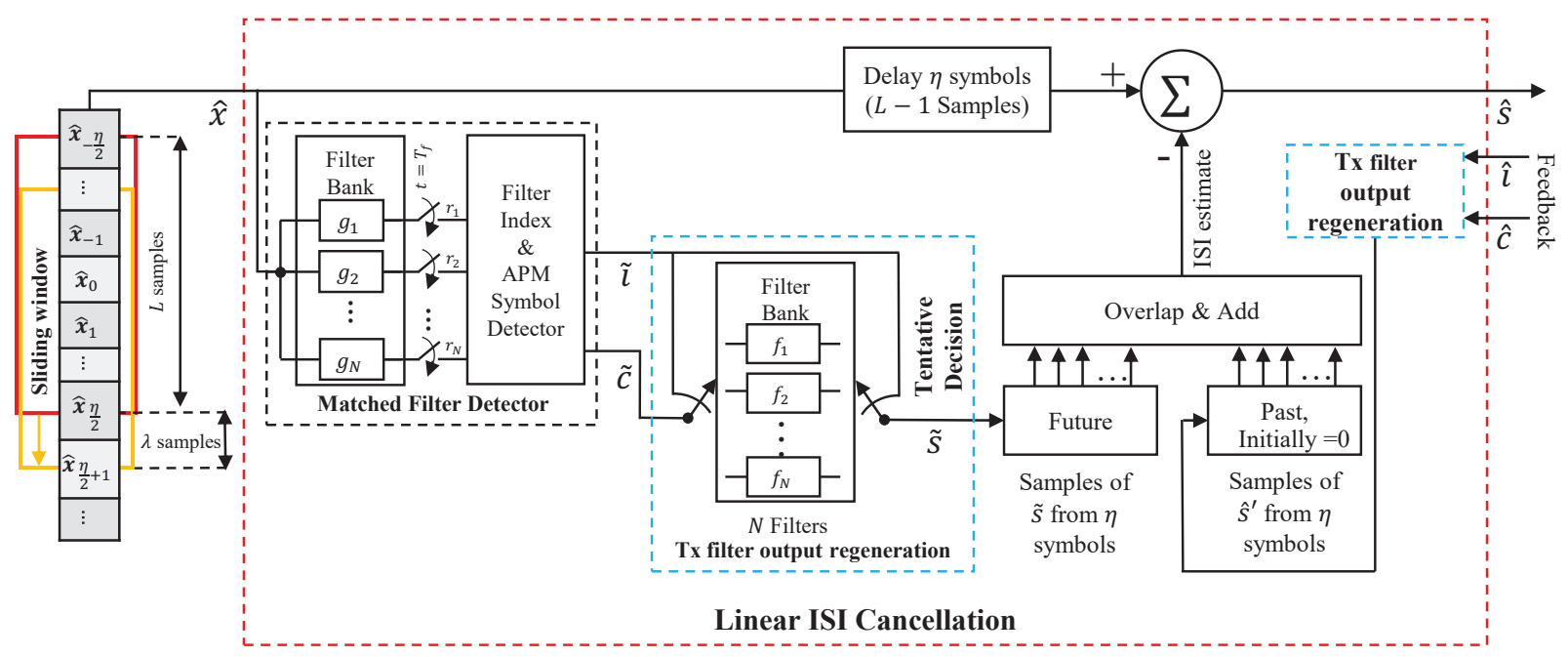

Fig. 3: ISI estimation and cancellation using feedback decisions $\{\hat{\imath}, \hat{c}\}$ for past symbols and tentative decisions $\{\tilde{\imath}, \tilde{c}\}$ for future symbols.

Considering an AWGN channel, equation (5) can be rewritten as follows:

$$
\begin{aligned}
y(t) & =x(t)+v(t)=\sum_{n} s_{n}\left(t-n \cdot T_{\text {sym }}\right)+v(t) \\
& =\sum_{n} c_{n} f_{i_{n}}\left(t-n \cdot T_{\text {sym }}\right)+v(t) .
\end{aligned}
$$

The received signal is sampled at the rate of $T_{s}$ and the $L$ received samples corresponding to APM symbol $c_{n}$ is given as follows:

$$
\begin{aligned}
y_{n}[m]= & \left(c_{n} \cdot f_{i_{n}}[m]\right. \\
& +\underbrace{\sum_{n^{\prime}>n} c_{n^{\prime}} \cdot f_{i_{n^{\prime}}}\left[m-\left(n^{\prime}-n\right) \lambda\right]}_{\text {anticausal ISI }} \\
& +\underbrace{\sum_{n^{\prime}<n} c_{n^{\prime}} \cdot f_{i_{n^{\prime}}}\left[m-\left(n^{\prime}-n\right) \lambda\right]}_{\text {causal ISI }}) \\
= & c_{n} \cdot v_{n}[m]
\end{aligned}
$$

where $f_{i_{n}}$ represents the $i^{t h}$ filter used to transmit the $n^{t h}$ symbol and $v_{n}[m]$ is the noise sampled at $t=n \cdot T_{\text {sym }}+m \cdot T_{s}$. Note that if the used pulse-shaping filters satisfy Nyquist condition for zero-ISI, then the received signal in AWGN channel is ISI free (ISI $=0$ in (12)) which is not the case in the proposed system.

Thus, the perfect ISI cancellation for the $L$ samples around any symbol requires the knowledge of the filter index and APM symbols for the $\eta$ past and $\eta$ future symbols (as shown in Fig. 2 where the ISI in the samples $\mathbf{I}_{3}^{0}$ of the first APM symbol $c_{0}$ can be eliminated by knowing all the future $\eta=6$ symbols). The proposed ISI cancellation algorithm takes the past $\eta$ detected APM symbols $\hat{c}_{n^{\prime}<n}$ along with their filter indices $\hat{\imath}_{n^{\prime}<n}$ as a feedback from the previous final decisions as shown in Fig. 1. However, a tentative decision as in [45] is required for the $\eta$ future symbols. Thus, the ISI cancellation and detection for the current symbol will be delayed by $\eta$ symbols after receiving the $L$ samples of $c_{n}$ as depicted in Fig. 3 in order to be able to mitigate the anticausal ISI. Note that the conventional transceiver delay is $d_{\text {conv. }}=\frac{\eta \cdot \lambda}{2}+\left\lfloor\frac{\lambda}{2}\right\rfloor$ samples, which corresponds to filter ramp-up delay and time needed to receive all $\lambda$ samples for first symbol. Thus, the additional FSIM system delay compared to conventional transceiver is $d_{\mathrm{FSIM}}=L+\eta \lambda-d_{\text {conv. }}=3 \frac{\eta \cdot \lambda}{2}+1-\left\lfloor\frac{\lambda}{2}\right\rfloor$ samples, which corresponds to the delay needed to receive the rest of the first $L$ samples and to eliminate the anticausal ISI from the $\eta$ future symbols.

The FSIM receiver with linear ISI cancellation is depicted in Fig. 3. The tentative decision of the APM symbol $\tilde{c}_{n^{\prime}>n}$ and the filter index $\tilde{\imath}_{n^{\prime}>n}$ for future $\eta$ symbols are detected by the matched filter as described in the subsection III-B2 using their corresponding $L$ samples of the received signal $y_{n^{\prime}}[m]$. These tentative decisions $\left\{\tilde{\imath}_{n^{\prime}}, \tilde{c}_{n^{\prime}}\right\}$ are used to regenerate the filter bank output originally generated at the transmitter side as $\tilde{s}_{n^{\prime}}[m]=\tilde{c}_{n^{\prime}} f_{\tilde{\imath}_{n^{\prime}}}[m]$. Similarly, this regeneration is performed for the past symbols using the feedback decisions $\left\{\hat{\imath}_{n^{\prime}}, \hat{c}_{n^{\prime}}\right\}$ to get $\hat{s}_{n^{\prime}}[m]=\hat{c}_{n^{\prime}} f_{\hat{\imath}_{n^{\prime}}}[m]$. As shown in Fig. 3 , the regenerated signals are saved in their corresponding registers and then used to generate the ISI estimation. Finally, the ISI cancellation is performed by subtracting the estimated ISI from the corresponding $L$ samples of the received signal $y_{n^{\prime}}[m]$. This aims to generate the estimated transmitter filter output $\hat{s}_{n}[m]$ for the APM symbol $c_{n}$ that can be expressed as follows:

$$
\hat{s}_{n}[m]=c_{n} f_{i_{n}}[m]+I S I_{\text {resid }}[m]+v_{n}[m],
$$

where $m=0, \ldots L-1$. The $I S I_{\text {resid }}[m]$ represents the residual ISI that might still persist due to the non-perfect ISI cancellation. Note that if the filters are well designed, the $I S I_{\text {resid }}[m]$ approaches zero. Finally, $\hat{s}_{n}[m]$ is passed through the filter index and the APM symbol detectors to recover the bitstreams $b_{1}$ and $b_{2}$ as described in Fig. 1.

\section{Filter Bank Design requirements}

Note that the proper design of the filter shape is crucial to allow the correct detection of the indexed filter. In this section, the filter bank design problem is presented, which is similar to the conventional pulse-shaping problem, but more 
constraints are added to maximize the correct detection of the selected filter index. The proposed system insists that ISI is not necessarily undesirable while it is controllable. The aim is to achieve better system capacity rather than enforcing zero interference.

The filter bank design problem is not a trivial task because all filters $f_{i}$ in the bank should be jointly designed to satisfy the following conditions and constraints:

1) $f_{i}$ is strictly band-limited with a bandwidth $B$ within the channel bandwidth $B_{c}$, where $B \leq B_{c}$ should be as large as possible. In addition, the filters $f_{i}$ should be around the center frequency of the channel (around the carrier frequency in the pass-band or around zero in the base-band).

2) All the filters should have an acceptable level of OutOf-Band (OOB) radiations with a feasible filter length. Thus, the Magnitude response $\left|F_{i}(\omega)\right|$ of all filters $\mathbf{f}_{i}$ should satisfy the following condition: $\left|F_{i}(\omega)\right| \leq$ $O O B_{\max }$ for $\omega \geq \omega_{s}$, where $\omega_{s}$ is the stop-band angular frequency. The bandwidth and OOB requirements can be expressed together by set of constraints to fit within a spectral mask.

3) Minimum cross-correlation between all normalized filters $f_{i}$ in the bank is required to maximize the correct filter index detection at the receiver side. The crosscorrelation between two filters $f_{i}$ and $f_{j}$ from the bank is given by:

$$
\begin{aligned}
& \left(f_{i}^{*} \star f_{j}\right)[d]=\sum_{l=-\infty}^{l=+\infty} f_{i}^{*}[l] \cdot f_{j}[l-d] \\
& \forall i, j=1,2, \ldots, N \text { and } d=0,2, \ldots, 2 L-1 .
\end{aligned}
$$

More precisely, the dot-product square between filters $\left\langle\mathbf{f}_{i}, \mathbf{f}_{j}\right\rangle^{2}$ should be minimized since the MF detector output taken at $t=T_{f}$ depends on this dot-product value.

4) The performance of FSIM depends on the ability of correct ISI estimation and cancellation and mainly on the correct tentative decisions in the presence of ISI. Thus, the introduced ISI distortion should be minimized while respecting the previous conditions and constraints, to avoid any error floor in terms of SER and BER.

5) The filter length $L$ should be kept the minimum possible to allow practical implementation. Hence, the tails of their impulse responses should decay to zero after a feasible number of symbols in order to have, after the time domain truncation to $\eta$ symbols, the best tradeoff filter length-OOB.

A straightforward solution for this filter design problem is to have orthogonal filters that occupy each different bandwidth by dividing $B_{c}$ into $N$ sub-bands. In this case, the first condition to have the same center frequency is not satisfied, which will lead to a lower SE when high $M$-ary schemes are used according to Table I. This case was discussed in [5] when applying IM in the frequency domain on the OFDM subcarriers. Hence, the frequency IM domain is a special case of the proposed filter index modulation without a noticeable SE enhancement. Finally, for a proper filter shape design, we have to minimize the injected ISI distortion from all filters (denoted by $I S I_{\text {metric }}$ ), while keeping the cross-correlation between all filter-shapes below a threshold $\epsilon$ and respecting a predefined spectral mask. Thus, this challenging filter bank design can be formulated as an optimization problem for a given filter length $L$ as follows:

$$
\begin{aligned}
\mathbf{P 1}: & \underset{\mathbf{f}_{1}, \ldots, \mathbf{f}_{N}}{\operatorname{minimize} I S I_{\text {metric }}} \\
& \\
& \text { subject to } \\
& \left|F_{i}(\omega)\right| \text { within a predefined spectral mask } \\
& \forall i \in\{1, \ldots, N\}, \\
& \left\langle\mathbf{f}_{i}, \mathbf{f}_{j}\right\rangle^{2} \leq \epsilon \\
& \\
& \left\|\mathbf{f}_{i}\right\|^{2}=1 \neq j, \forall i \text { and } \forall j \in\{1, \ldots, N\}, \\
& \forall i \in\{1, \ldots, N\} .
\end{aligned}
$$

Such a problem is not tackled previously in the literature where $N$ filter shapes are to be designed jointly with similar constraints. This problem will be properly addressed and discussed in our future publication due to the page limitation.

\section{Theoretical Performance Analysis}

In this section, the probability of error for the filter index is evaluated, then the SER and the total BER is deduced to truly characterize the FSIM system performance in AWGN channel. Note that both proposed detectors (MF and joint ML) achieve similar performance (negligible difference), as it will be shown in the next section. For this reason, the low complexity MFbased detector is only considered in the following theoretical derivations.

The probability of error for filter index pilots the system performance because the error in filter detection leads to an additional ISI, and thus more errors can appear in the detection of APM symbols.

For each symbol period, the input to matched filter detector is the $L$ samples as expressed in (13). In the following, we will assume that the filter bank is well designed where the ISI is perfectly canceled (i.e., $I S I_{\text {resid }}=0$ ). Hence, the probability of error that will be derived can be considered the lower bound of the proposed system.

The output of the matched filter bank sampled at $t=T_{f}$ given by $r_{k}$ in (9) can be rewritten as:

$$
\begin{aligned}
r_{k} & =\left\langle\mathbf{f}_{k}, \mathbf{f}_{i_{n}}\right\rangle \cdot c_{n}+\left\langle\mathbf{f}_{k}, \mathbf{v}_{n}\right\rangle \\
& = \begin{cases}c_{n}+\left\langle\mathbf{f}_{k}, \mathbf{v}_{n}\right\rangle & \text { for } k=i_{n} \\
\left\langle\mathbf{f}_{k}, \mathbf{f}_{i_{n}}\right\rangle \cdot c_{n}+\left\langle\mathbf{f}_{k}, \mathbf{v}_{n}\right\rangle & \text { for } k \neq i_{n}\end{cases}
\end{aligned}
$$

Note that when the $N$ filters are orthogonal $\left\langle\mathbf{f}_{k}, \mathbf{f}_{i}\right\rangle=0$, the system will be transformed into frequency band indexation, which results in a limited SE enhancement. However, in our proposed system, the filters are not orthogonal because they occupy the same bandwidth, and they have the same center frequency to target a more significant SE enhancement.

To evaluate the probability of error, let us suppose that the filter $f_{1}$ is selected at the transmitter side for the APM symbol $c_{n}$. Then, the vector $\mathbf{r}_{k}$ composed of the elements $r_{k}$ for $k=$ $1, \ldots, N$ is given as follows:

$$
\mathbf{r}_{k}=\left[c_{n}+v_{1},\left\langle\mathbf{f}_{2}, \mathbf{f}_{1}\right\rangle \cdot c_{n}+v_{2}, \ldots,\left\langle\mathbf{f}_{N}, \mathbf{f}_{1}\right\rangle \cdot c_{n}+v_{N}\right],
$$


where $v_{k}=\left\langle\mathbf{f}_{k}, \mathbf{v}_{n}\right\rangle$ are complex valued zero-mean mutually statistically independent Gaussian random variables with equal variance $\sigma^{2}=\sigma_{k}^{2}=\frac{1}{2} N_{0}$, where $N_{0}$ is the noise spectral density [46]. According to (10), the detection of the filter index selects the maximum $U_{k}=\left\|r_{k}\right\|^{2}$ which represents the energy of $r_{k} . U_{k}$ is described as a statistically independent non-central chi-square distribution [46] for all $k$, each having 2 degrees of freedom where the non-centrality parameter $\alpha^{2}$ with the energy of APM symbol $\mathcal{E}_{q}$ is given by:

$$
\alpha_{k, q}^{2}= \begin{cases}\left\|c_{n}\right\|^{2}=\mathcal{E}_{q} & \text { for } k=1 \\ \left\langle\mathbf{f}_{k}, \mathbf{f}_{1}\right\rangle^{2} \cdot\left\|c_{n}\right\|^{2}=\left\langle\mathbf{f}_{k}, \mathbf{f}_{1}\right\rangle^{2} \cdot \mathcal{E}_{q} & \text { for } k \neq 1,\end{cases}
$$

where $\mathcal{E}_{q}$ is the energy of the transmitted APM symbol $c_{n}$. Thus, we obtain the PDF of $U_{k}$ as

$$
p\left(u_{k}\right)=\frac{1}{2 \sigma^{2}} e^{-\frac{u_{k}+\alpha_{k}^{2}}{2 \sigma^{2}}} \mathcal{I}_{0}\left(\frac{\alpha_{k} \sqrt{u_{k}}}{\sigma^{2}}\right),
$$

where $u_{k} \geq 0$ and $\mathcal{I}_{0}(\rho)$ is the zero order modified Bessel function of $1^{\text {st }}$ kind given by [46]:

$$
\mathcal{I}_{0}(\rho)=\sum_{\beta=0}^{\infty} \frac{(\rho / 2)^{2 \beta}}{(\beta !)^{2}} \rho \geq 0 .
$$

The error probability of the filter index can be deduced by deriving the probability that the detector makes a correct index decision. This is the probability that $u_{1}$ is larger than all other $N-1 u_{k}$ values for $k \neq 1$. This probability of correct decision for a given APM symbol energy $\mathcal{E}_{q}$ may be expressed as:

$$
\begin{aligned}
P_{c, \mathcal{E}_{q}} & =P\left(U_{2}<U_{1}, U_{3}<U_{1}, \ldots, U_{N}<U_{1}\right) \\
& =\int_{0}^{\infty} P\left(U_{2}<U_{1}, \ldots, U_{N}<U_{1} \mid u_{1}\right) \cdot p\left(u_{1}\right) d u_{1}
\end{aligned}
$$

where $P\left(U_{2}<U_{1}, U_{3}<U_{1}, \ldots, U_{N}<U_{1} \mid U_{1}\right)$ denotes the joint probability that $u_{2}, u_{3}, \ldots u_{N}$ are all less than $u_{1}$ conditioned on a given $u_{1}$. Then this joint probability is averaged over all $u_{1}$. The filters are considered orthogonal when deriving the lower bound, thus these $N-1$ variables $u_{k}$ becomes statistically independent under this assumption with chi-square distribution $\left(\alpha_{k, q}^{2}=0\right.$ for $\left.k \neq 1\right)$. Since the $u_{k}$ are statistically independent, the joint probability can be factorized as a product of $N-1$ marginal probabilities of the form:

$$
P\left(u_{k}<u_{1} \mid u_{1}\right)=\int_{0}^{u_{1}} p_{u_{k}}\left(x_{k}\right) d x_{k}, \quad k=2,3, \ldots, N .
$$

Thus, the probability of a correct decision is given by:

$$
P_{c, \mathcal{E}_{q}}=\int_{0}^{\infty}\left(\prod_{2}^{N} P\left(u_{k}<u_{1} \mid u_{1}\right)\right) \cdot p\left(u_{1}\right) d u_{1}
$$

and the probability of a filter index error with a given APM symbol energy $\mathcal{E}_{q}$ is as follows:

$$
P_{e, \mathcal{E}_{q}}=1-P_{c, \mathcal{E}_{q}} .
$$

Hence, the average probability of filter index error is the weighted average of filter error over the $Q$ possible energy levels of APM symbols, given as follows:

$$
P_{e, \text { filter }}=\sum_{q=1}^{Q} P_{e, \mathcal{E}_{q}} \cdot P\left(\mathcal{E}_{q}\right),
$$

where $P\left(\mathcal{E}_{q}\right)$ is the probability of occurrence of the energy level $\mathcal{E}_{q}$. For example, when the used APM is 16-QAM, there are $Q=3$ possible energy levels $\mathcal{E}_{q}=[0.2,1,1.8]$ and their probability of occurrence is $P\left(\mathcal{E}_{q}\right)=[0.25,0.5,0.25]$ if all APM symbols are equiprobable.

An erroneous detection of a filter index leads to the attempt of APM demodulation using the signal output of an mismatched filter with more ISI most probably. Thus, the total probability of APM symbol error $P_{e, A P M}$ in FSIM system can be written according to the law of total probability as follows:

$$
\begin{aligned}
P_{e, A P M}= & P_{(A P M \text { error } \cap \text { correct filter })} \\
& +P_{(A P M \text { error } \cap \text { false filter })} \\
= & \left.\left(1-P_{e, f i l t e r}\right) P_{(A P M} \text { error } / \text { correct filter }\right) \\
& +P_{e, \text { filter }} P_{(A P M \text { error } / \text { false filter }),}
\end{aligned}
$$

where $P_{A P M \text { error/correct filter }}\left(P_{A P M \text { error/false filter }}\right)$ is the probability of APM symbol error knowing that the filter index is correctly estimated (mis-detected). Hence, we are deriving the lower bound under the perfect ISI cancellation assumption, then the probabilities of APM symbol error in both cases are equal to the probability of APM symbol error in conventional transceiver with Nyquist filters.

Consequently, the correct FSIM symbol detection occurs when both the filter and the APM symbol are correctly estimated, then the probability of a correct FSIM decision is $\left(1-P_{e, f i l t e r}\right)\left(1-P_{e, A P M}\right)$ and the SER is given by:

$$
S E R=1-\left(1-P_{e, \text { filter }}\right)\left(1-P_{e, A P M}\right) \text {. }
$$

The average probability of virtual (real) bit error can be deduced from filter (APM symbol) error similar to [46, p. 262 (5-2-24)], thus they can be expressed respectively as follows:

$$
\begin{aligned}
P_{b, \text { filter }} & =\frac{N}{2(N-1)} P_{e, \text { filter }} \\
P_{b, A P M} & =\frac{M}{2(M-1)} P_{e, A P M},
\end{aligned}
$$

Similarly to SER, the total BER is deduced but while taking into consideration the weight of real and virtual bits:

$$
B E R=\frac{\log _{2} N}{\mathcal{L}_{\mathrm{FSIM}}} P_{b, \text { filter }}+\frac{\log _{2} M}{\mathcal{L}_{\mathrm{FSIM}}} P_{b, A P M} .
$$

Finally, we should mention that the cross-correlation between all filters affects the proposed system's performance and should be minimized to enhance the system performance. Besides, the introduced ISI by all filters should also be minimized to approach the lower bound of the error probability for the filter index that affects the APM symbols' detection. This probability confirms that the proposed system's success is based on the filter bank design where the cross-correlation of these filters and their introduced ISI should be jointly minimized. Finally, these theoretical lower bound for error probabilities of filter index detection and BER are validated in the next section.

\section{Numerical Results Analysis and Discussions}

In this section, firstly, the theoretical error probability of filter index detection (25) using the MF-based detector is 


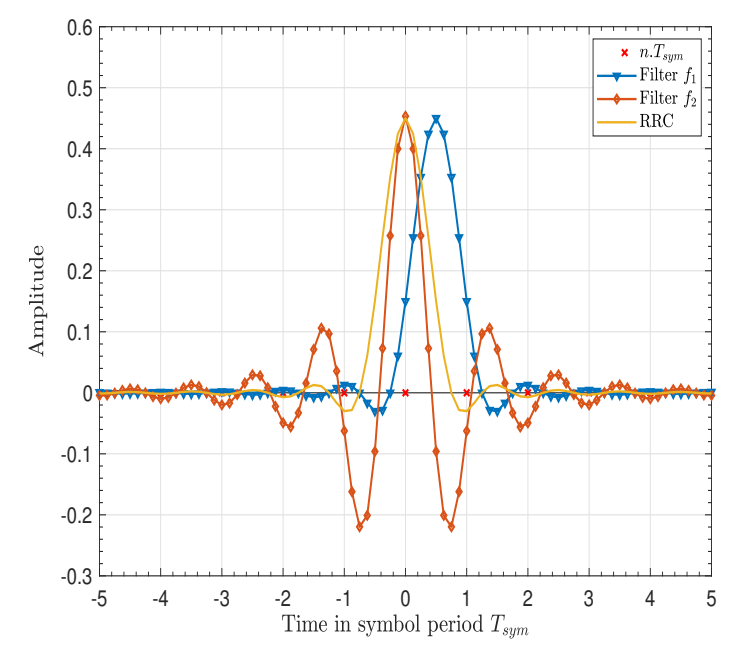

Fig. 4: Impulse Response for the two filters used in our simulations compared to RRC pulse-shaping filter, where $\eta=10$ and $\lambda=8$.

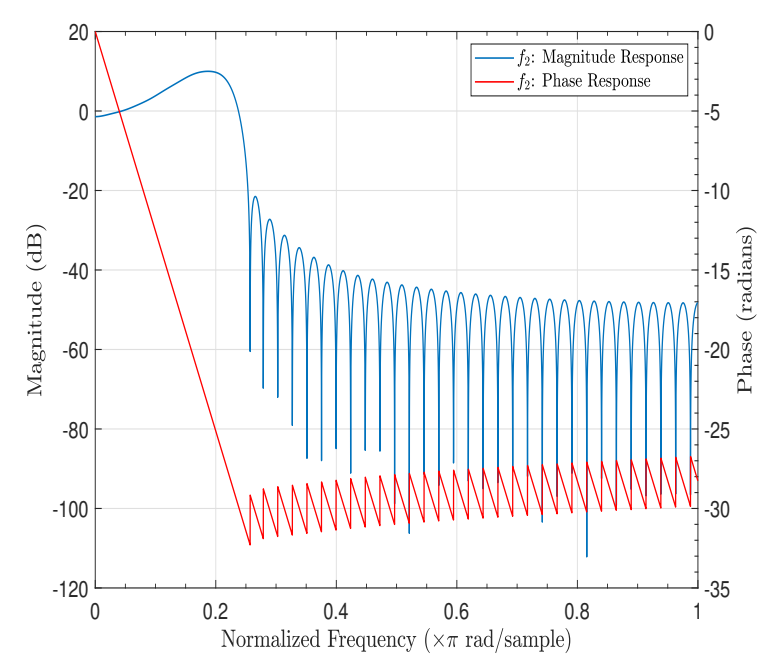

Fig. 6: Magnitude and phase response of the filter $f_{2}$ depicted in Fig. 4.

evaluated and compared to the numerical results obtained with Monte Carlo simulations under different configurations. Secondly, the performance of the proposed system is evaluated when a joint ML and an MF-based detector are employed and compared at different transmission rates to the theoretical lower bound (30) and to the performance of the equivalent APM scheme of the same SE in AWGN channel and a multipath frequency-selective fading channel.

It is worth mentioning that the filter bank design of $N$ filter shapes is not a trivial problem with these new constraints and conditions. For this reason, we designed 2 and 4 nonoptimal filter shapes to prove the feasibility of the proposed scheme and to illustrate the minimum performance gain and SE enhancement that can be achieved. The impulse responses of the two filters are presented in Fig. 4, and their magnitude and phase responses are shown in Fig. 5 and 6. Note that the dot product of these filters is $\left\langle\mathbf{f}_{1}, \mathbf{f}_{2}\right\rangle=0.2057$. Moreover, the complete filter responses at the receiver, after correct matched

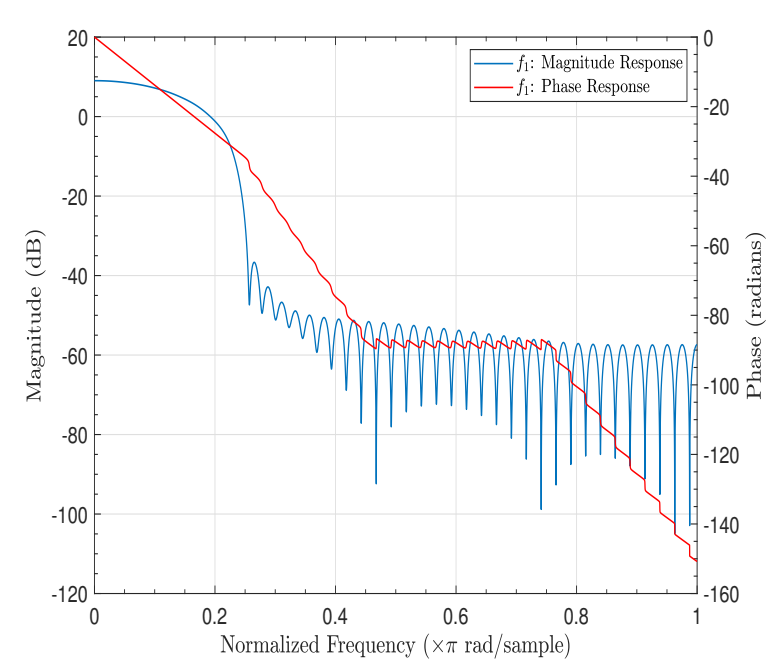

Fig. 5: Magnitude and phase response of the filter $f_{1}$ depicted in Fig. 4.

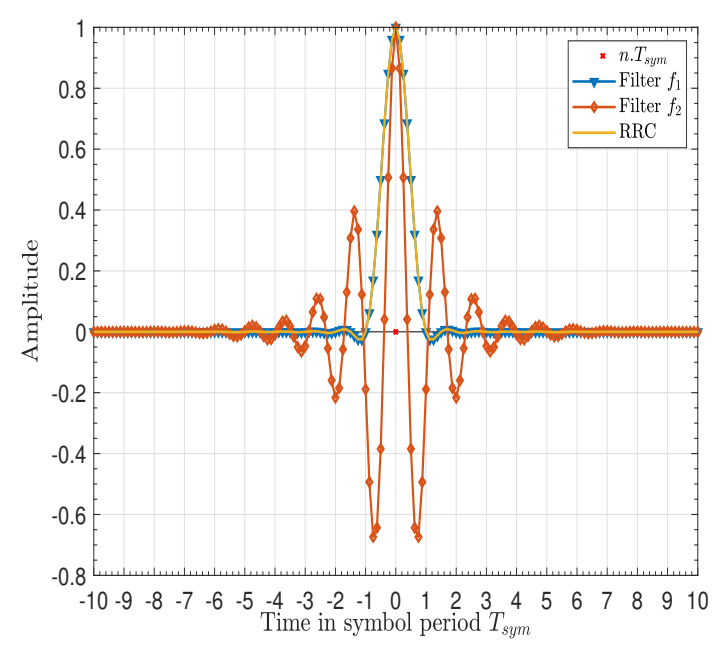

Fig. 7: Complete responses of the 2 filters obtained after correct matched filtering at the receiver side, where $\eta=10$ and $\lambda=8$.

filtering $\left(\mathbf{f}_{i} * \mathbf{g}_{i}\right)$ are depicted in Fig. 7. The introduced ISI clearly appears in $f_{2}$ because the result of $\mathbf{f}_{2} * \mathbf{g}_{2}$ doesn't have a zero-crossing at $t=n . T_{\text {sym }}$ for all $n$.

The proposed system is studied at different transmission rates (3 to 7 bits/symbol) by using different numbers of filter shapes and different QAM orders. The used simulation parameters are summarized in Table II.

TABLE II: Simulation parameters

\begin{tabular}{||c|c||}
\hline Parameters & Value \\
\hline \hline$M$-ary QAM & {$[4,8,16,32,64]$} \\
\hline$N$ & {$[2,4]$} \\
\hline$\eta$ & 10 \\
\hline Oversampling factor: $\lambda$ & 8 \\
\hline Number of symbols & $5 \times 10^{5}$ \\
\hline $\begin{array}{c}\text { Pulse-shaping filter } \\
\text { for Conventional Transceiver }\end{array}$ & $\begin{array}{c}\text { Root Raised Cosine } \\
\text { (RRC) }\end{array}$ \\
\hline
\end{tabular}




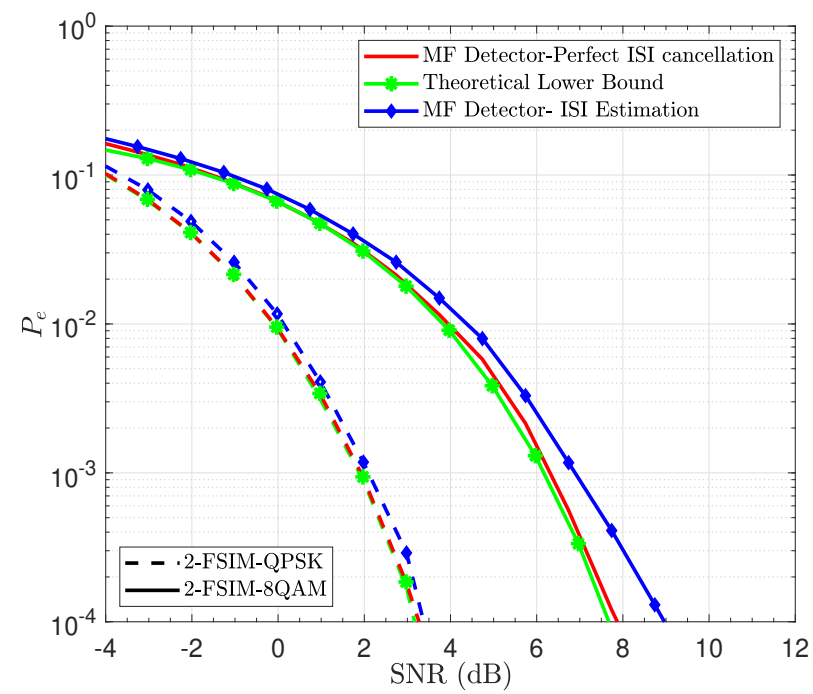

(a)

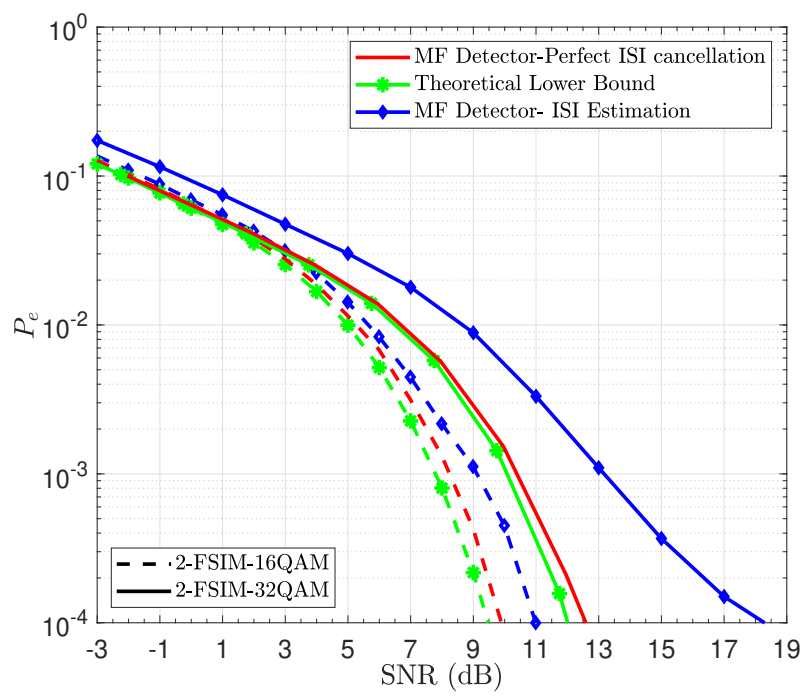

(b)

Fig. 8: The error probabilities of filter index detection with ISI estimation and cancellation, perfect ISI cancellation, and the derived theoretical lower bound of 2-FSIM-MQAM $(N=2$ ). The SE is: (a) $[3,4]$ bits/symbol using $M=[4,8]$, (b) $[5,6]$ bits/symbol using $M=[16,32]$.

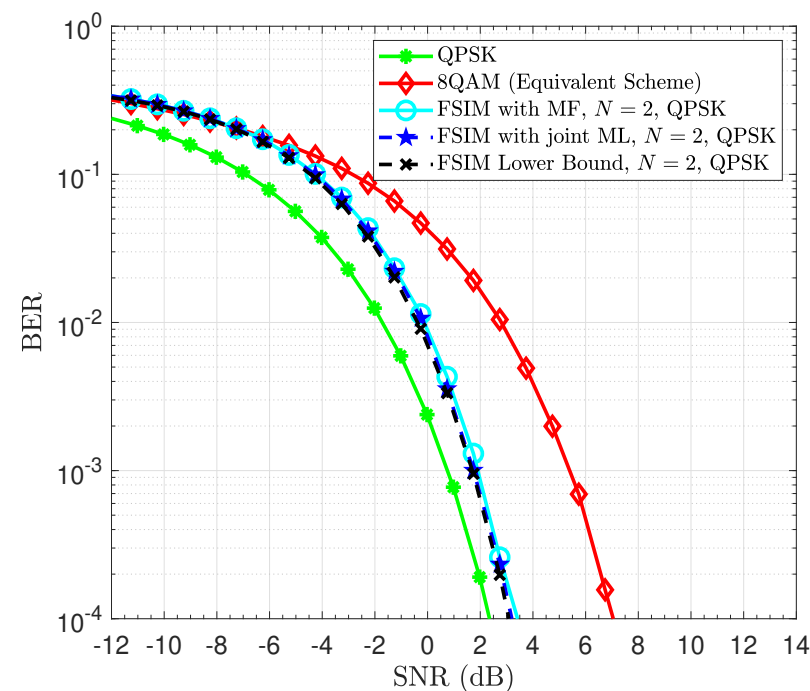

(a)

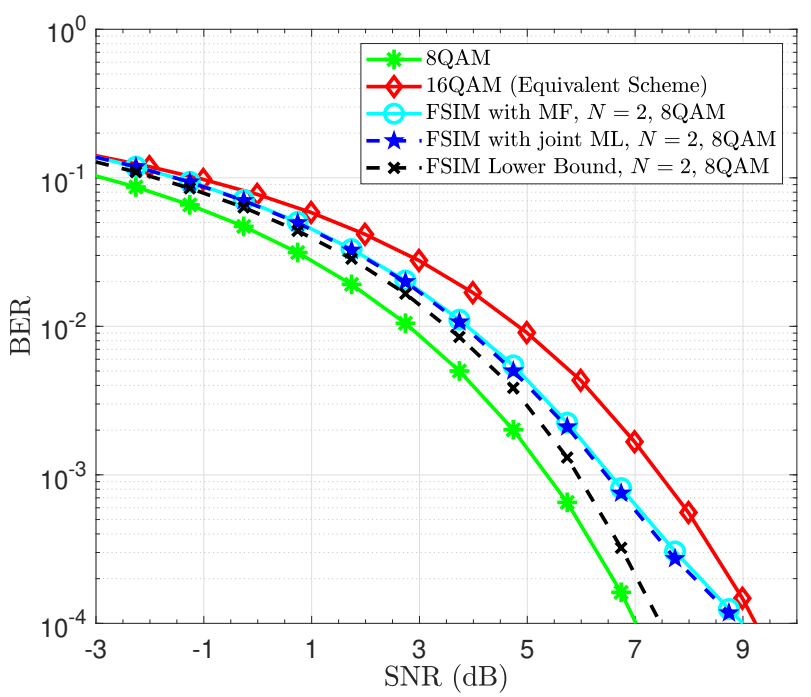

(b)

Fig. 9: Uncoded BER performance of 2-FSIM- $M$ QAM, its analytical lower bound and its equivalent scheme $2 M Q A M$ of the same SE: (a) 3 bits/symbol using $M=4$, (b) 4 bits/symbol using $M=8$.

Figure $8 \mathrm{a}$ and $8 \mathrm{~b}$ compare the theoretical lower bound (25) and simulated probabilities of error of filter index detection with perfect ISI cancellation, and with ISI estimation and cancellation as described in subsection III-B3. Note that perfect ISI cancellation means that the tentative decisions for filter index and APM symbol are correctly detected, but those decisions for past symbols are fed back after the final detection. This comparison is performed with $N=2$ filters and different $M$-ary QAM where the notation $N$-FSIM-MAPM is adopted for our proposed system's performance analysis.

It can be observed from Figs $8 \mathrm{a}-8 \mathrm{~b}$ that the theoretical lower bound (25) derived under the assumption of perfect ISI cancellation and orthogonal filters matches the simulation results of perfect ISI cancellation with a small difference due to non-orthogonal filters (dot-product is 0.2057), and thus the theoretical lower bound is validated. In addition, the performance of 2-FSIM-QPSK with ISI estimation is near the optimal ISI cancellation as shown in Fig. 8a, because QPSK can support a high ISI level without leading to a false detection (high minimum distance $d_{\min }$ between constellations), so a better ISI estimation and cancellation are achieved. Similarly, this probability with ISI estimation and cancellation using 2FSIM-8QAM and 2-FSIM-16QAM is very tight to the lower bound at low Signal-to-noise Ratio (SNR) values, while the gap between them increases at high SNR values due to the residual ISI. In addition, it is clear that this degradation due to residual ISI is more important with higher $M$-ary QAM as shown with 2-FSIM-32QAM in Fig. 8b. This can be justified by the fact that higher $M$-ary QAM schemes are more sensitive to ISI (smaller $d_{\min }$ ) compared to QPSK. Note that the two used filter shapes satisfy all the filter bank design requirements mentioned in sub-section III-C, but they 


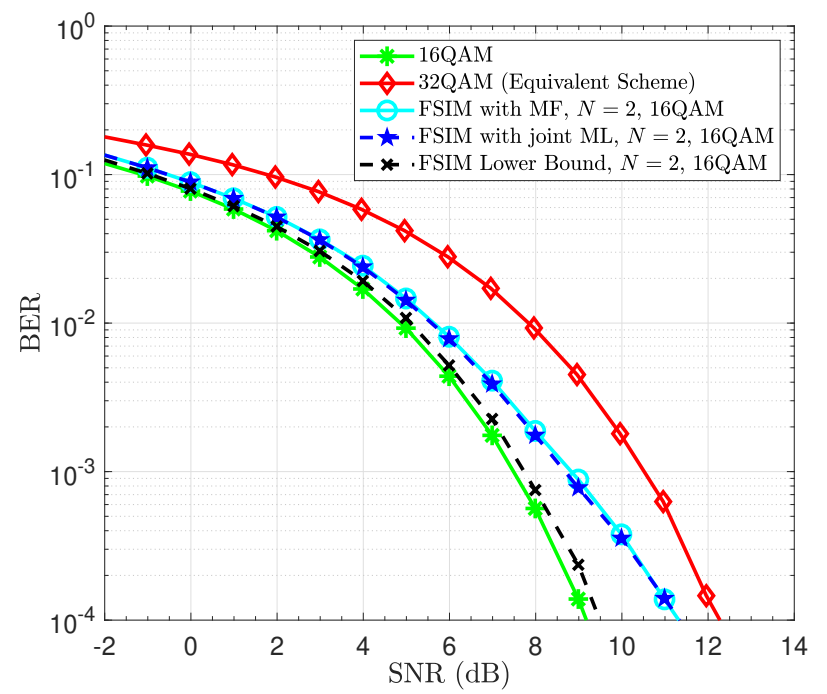

(a)

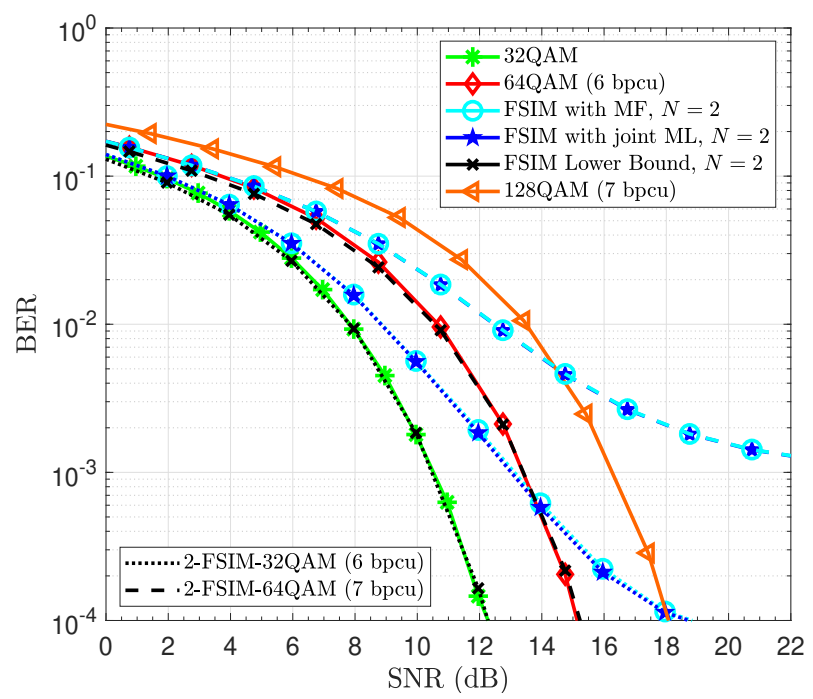

(b)

Fig. 10: Uncoded BER performance of 2-FSIM-MQAM, its analytical lower bound and its equivalent scheme $2 M$ QAM of the same SE: (a) 5 bits/symbol using $M=16$, (b) 6 and 7 bits/symbol using $M=32$ and $M=64$ respectively.

are not the optimal filters. Thus, the performance of FSIM can become tighter to the lower bound when the filter's bank shapes are optimally designed. Hence this analytical bound is a helpful indicator for evaluating the performance of the proposed system.

In the following, the proposed FSIM system is compared to the conventional transceivers under different configurations but with the same SE. The BER of these systems as a function of the average SNR is evaluated using Monte Carlo Simulations and theoretical lower bound according to (30). The comparison in Figs. 9a-10b is performed with ISI estimation and cancellation under an AWGN channel with MF and joint ML detectors. It should be highlighted that the theoretical BER lower bound (30) and the simulated BER with perfect ISI cancellation are very tight, and they are represented by a single curve named FSIM lower bound in Fig. 9a-10b. In addition, these figures show that the performance with the MF detector is very tight to that with the joint ML detector but with extremely lower complexity. The propagation of error issue clearly appears in Fig. 10b using both detectors (or any other) due to residual ISI from the error in tentative and feedback decisions in the preceding block for ISI estimation and cancellation. Thus, the error propagation is an issue with any detector, and it can be minimized by optimal filter design that allows mainly the correct tentative decisions.

In order to conduct a fair comparison, the performance of the proposed system is compared to an equivalent conventional transceiver ( $M$-ary QAM scheme with RRC filter) of the same SE. The system 2-FSIM-QPSK is depicted in Fig. 9a, where an important SNR gain of $3.8 \mathrm{~dB}$ is obtained at BER $=10^{-4}$ compared to the equivalent 8QAM scheme. Accordingly, for the same BER performance, the SE enhancement by 1 bit/symbol requires an additional $0.9 \mathrm{~dB}$ only to the required SNR of QPSK. However, for the same SE enhancement with respect to $8 \mathrm{QAM}$ and $16 \mathrm{QAM}$, an additional $2 \mathrm{~dB}$ is required with 2-FSIM-8QAM and 2-FSIM-16QAM as shown in Figs. $9 \mathrm{~b}$ and 10a respectively. While the higher-order equivalent schemes as 16QAM and 32QAM need an additional $2.2 \mathrm{~dB}$ and $3.1 \mathrm{~dB}$ respectively instead of $2 \mathrm{~dB}$ with 2-FSIM-8QAM and 2-FSIM-16QAM. It is worth mentioning that a higher SNR gain is achieved when the proposed system, using two filters, is applied to a square QAM, so this system allows us to avoid the inherited performance degradation of non-square QAM by using FSIM with the lower square $M$-ary QAM order. In other words, as depicted in Fig. 9b the system 2-FSIM-8QAM has a smaller SNR gain due to the fact that the SNR gap between the performance of the non-square constellations of $8 \mathrm{QAM}$ and that of 16QAM is small for the same BER performance.

It is worth mentioning that these FSIM schemes are able to achieve SE and EE gain even with non-optimal filters compared to conventional APM schemes. In addition, this superiority is maintained when the FSIM is compared to existing single and dual-mode SISO-IM schemes of worse EE due to the need of higher power to maintain the same SE by using larger $M$-ary and/or to detect the transmitted VBs (see [43] and references therein).

For further analysis of the proposed scheme, the results with higher-order APM as 2-FSIM-32QAM and 2-FSIM-64QAM are presented in Fig. 10b, where their performance with ISI estimation and cancellation starts to degrade compared to equivalent schemes 64QAM and 128QAM respectively at high SNR values. It can be noticed that at low SNR, the performance of the proposed systems with high $M$-ary APM is better than their equivalent schemes. An error floor appears with the higher-order $M$-ary APM caused by the residual ISI not completely mitigated by the receiver, and which has been induced by the non-optimal filters being used.

However, the BER of the latter two schemes with perfect ISI cancellation is very tight to the performance of 32QAM and 64QAM, respectively, while enhancing the transmission rate by 1 bit. Hence, this maximum achievable performance shows that there is always room for future improvements by designing an optimal filter bank and proposing better ISI mitigation techniques. 


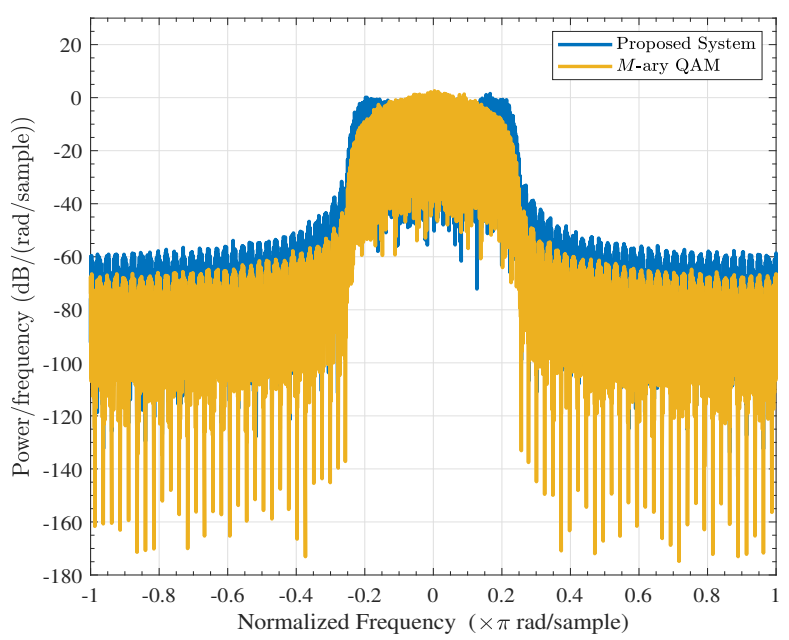

Fig. 11: Spectrum comparison between the proposed FSIM system with 2 non-optimal filters and the conventional transceivers with QAM and RRC filters.

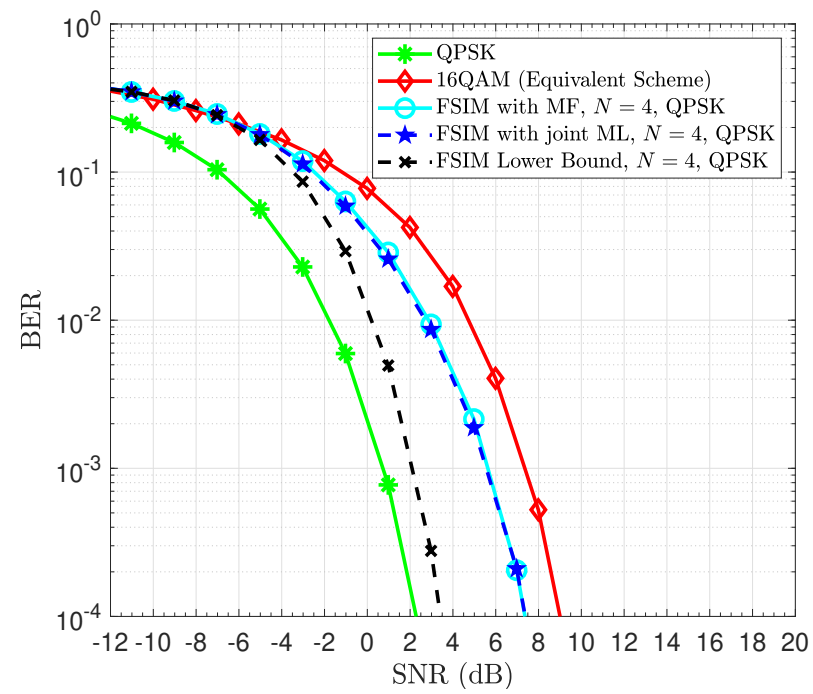

(a)

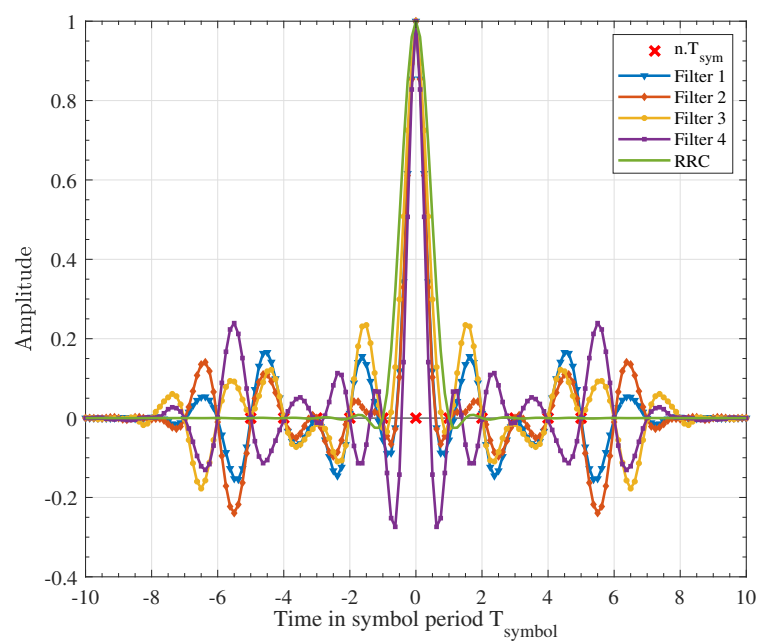

Fig. 12: Complete responses of the 4 filters obtained after correct matched filtering at the receiver side, where $\eta=10$ and $\lambda=8$.

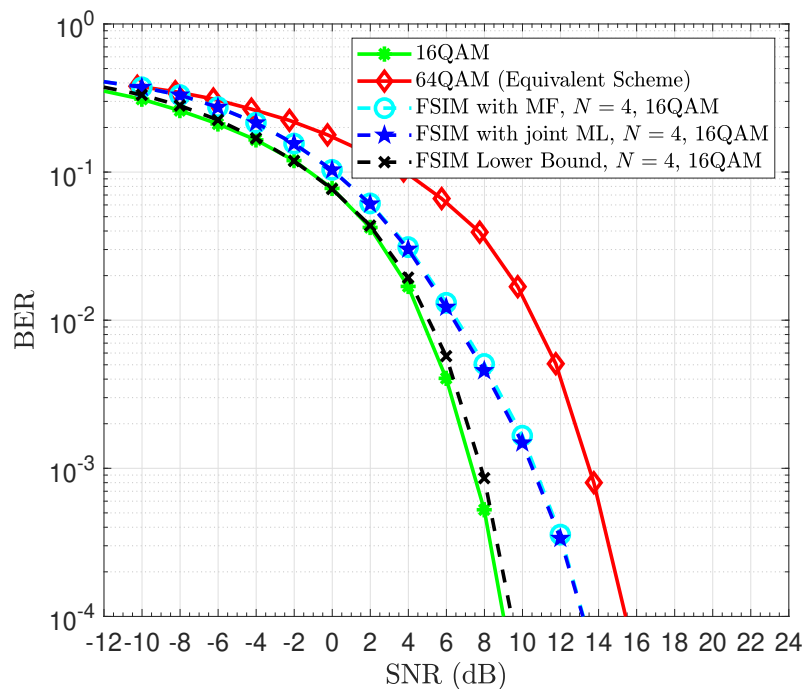

(b)

Fig. 13: Uncoded BER performance of 4-FSIM-MQAM, its analytical lower bound and its equivalent scheme $4 M$ QAM of the same SE: (a) 4 bits/symbol using $M=4$, (b) 6 bits/symbol using $M=16$.

The spectrum of the conventional transceiver and the 2FSIM-MQAM are compared in Fig. 11. It can be observed that most of the emitted power of both systems are concentrated on the same bandwidth. However, the OOB radiations of the proposed system is $10 \mathrm{~dB}$ higher than that of a conventional transceiver due to the fact the used filter $f_{2}$ has higher OOB radiation compared to $f_{1}$ and $\mathrm{RRC}$ filters.

By considering the filter bank design requirements mentioned in sub-section III-C, a set of $N=4$ filters is designed by using $\eta$ and $\lambda$ as mentioned in Table II, and the complete filter response is depicted in Fig. 12. The mean of the signal to the introduced ISI power ratio for all possible filter sequence permutations is $26.29 \mathrm{~dB}$, and the dot-product symmetric matrix between these non-optimal filters is:

$$
\boldsymbol{\Xi}=\left[\begin{array}{cccc}
1 & 0.7615 & 0.7284 & 0.334 \\
0.7615 & 1 & 0.6376 & 0.0887 \\
0.7284 & 0.6376 & 1 & 0.67 \\
0.334 & 0.0887 & 0.67 & 1
\end{array}\right]
$$

The results using these 4 filters with QPSK and 16QAM is depicted in Fig. 13a and Fig. 13b, where 4-FSIM-QPSK (4-FSIM-16QAM) has $1.7(2.2) \mathrm{dB}$ gain compared to its equivalent scheme 16QAM (64QAM). Note that 4-FSIMQPSK (4-FSIM-16QAM) requires 3.9 (3.6) dB more than its lower bound due to high dot-product between some filters.

After presenting these encouraging FSIM scheme results, its BER vs $E_{s} / N_{0}$ ( $E_{s}$ is the energy per symbol) is evaluated and compared in a frequency selective fading channel to 


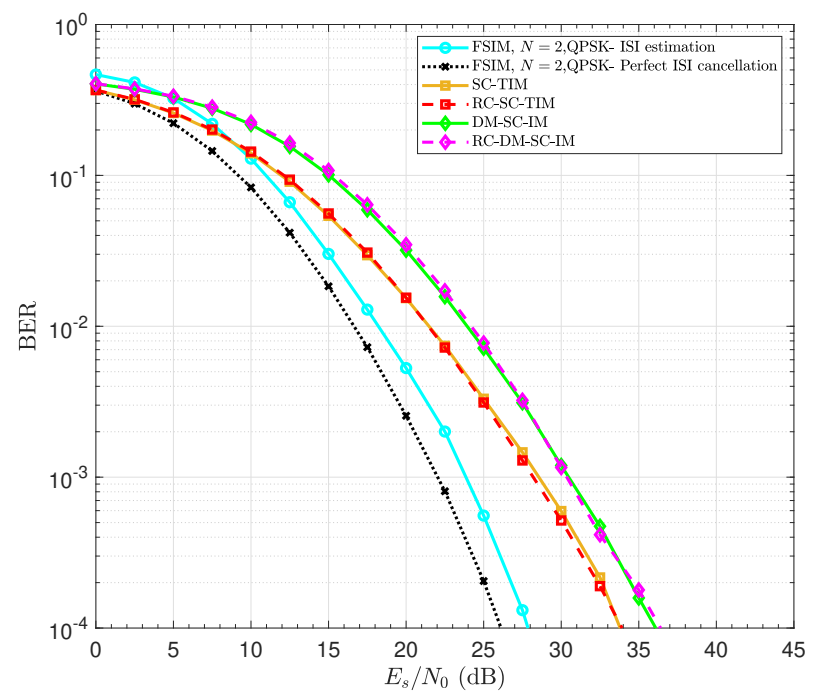

(a)

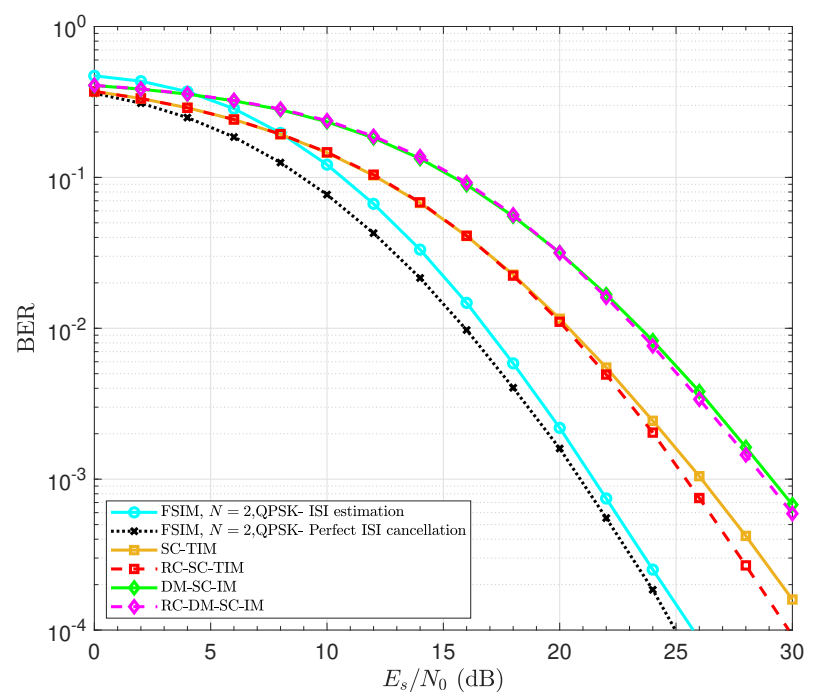

(b)

Fig. 14: Average BER comparison of different SC schemes with IM in a frequency selective Rayleigh fading: (a) $J=2$, (b) $J=4$.

the competitors SC schemes in single and dual-mode. A Rayleigh fading channel is considered with $J$-paths of an integer multiple of symbol period delays, and the MMSEFDE is used for all schemes of the same SE (DM-SC-IM, SC-TIM and their Reduced Correlation (RC) versions with interleaver [6]). Note that the optimal joint ML detector is used for these schemes, while a MF-based detector of lower complexity is used for FSIM. The frame setup in symbol length is: $N_{s}=1015, N_{Z P}=N_{C P}=9$, and the schemes' configuration, to have average $\mathrm{SE} \approx 3$ bits/symbol, are: (RC)SC-TIM: $M=16, N_{a}=3, N_{g}=5, G=203$, (RC-)DM-SCIM: $M_{A}=8, M_{B}=4, N_{a}=2, N_{g}=5, G=203$, and FSIM: $M=4, N=2$. Figs. $14 \mathrm{a}-14 \mathrm{~b}$ show clearly that FSIM with ISI estimation and cancellation outperforms all other schemes with a minimum gain of $6 \mathrm{~dB}(4.2 \mathrm{~dB})$ compared to the secondbest scheme RC-SC-TIM in Rayleigh selective channel with $J=2(J=4)$.

Furthermore, we would like to mention that the proposed system can achieve better performance and a higher EE gain by using an optimal filter bank satisfying the requirement discussed in Section III-C. Note that the design of a larger filter bank $(N>4)$ is a challenging and open research problem that goes beyond the scope of this work, and solving this problem will certainly lead to better SE gain.

Finally, to avoid any ambiguity between the filter IM domain and existing IM schemes, it is interesting to highlight the differences between the proposed filter domain and the Media-Based Modulation (MBM) IM scheme [47]. MBM uses different radiation patterns to convey the information bits using $N_{R F \text { mirrors }}$ RF mirrors after the transmit antenna with a single RF chain to generate different fade realizations. Therefore, MBM can achieve $N_{R F}$ mirrors bits/symbol as long as the independence of the generated radiation patterns is guaranteed, similar to Extended Space Shift Keying [48] with $N_{t}$ Tx antennas and RF chains in the spatial IM domain. Consequently, MBM requires a rich scattering environment to allow the detection of the different fade realizations, whereas FSIM is not limited to any channel condition. Thus, MBM faces a challenging problem to design distinguishable radiation patterns similar to FSIM. In contrast to FSIM, the main shortcomings of the MBM system is the excessive channel sounding burden and pilots/training sequence overhead to estimate the $2^{N_{R F} \text { mirrors }}$ fade realizations for a single $\mathrm{Rx}$ antenna. In addition, MBM is a linear time-varying system that can expand the spectrum [49], and it also suffers from spectral regrowth due to radiation switching between different signaling intervals. Thus, the achievable SE for MBM is degraded in contrast to FSIM and filter IM domain in general. Moreover, MBM performance gain is achieved only where more than one Rx antenna is used (SIMO or MIMO) [50], whereas FSIM can achieve an important gain even using nonoptimal filters in SISO mode.

\section{CONCLUSION}

Index Modulation is explored in the spatial, temporal, and frequency domain. In this paper, we propose a novel domain for IM, named the "Filter domain" and a novel scheme "Filter Shape Index Modulation" where the index of the filter is used at the transmitter to convey additional information bits and enhance the SE by $\log _{2} N$. Note that this domain allows a SE enhancement by at least $1 \mathrm{bit} / \mathrm{s} / \mathrm{Hz}$ in contrast to the existing domains in SISO (temporal and frequency domains) where their maximum enhancement is limited to $0.6 \mathrm{bit} / \mathrm{s} / \mathrm{Hz}$ or less.

This novel domain is investigated by proposing the FSIM system, where a bank with different filter shapes is used. In addition, the joint ML detector that detects the filter index and the transmitted APM symbol is presented. Then, a low complexity optimal detection scheme based on a Matched filter is proposed. Moreover, an ISI cancellation process is proposed due to the fact that FSIM introduces a controlled ISI. Note that the proposed system can be reconfigured to act as a conventional transceiver by using the same Nyquist filter for all symbols.

The theoretical lower bounds for filter error probability and BER are derived, and the analysis validates the achieved performance using the proposed FSIM approach. Monte Carlo 
simulations showed that the FSIM-QPSK system with 2 and 4 non-optimal filters offers, at $\mathrm{BER}=10^{-4}$ and same $\mathrm{SE}$, a gain of $3.8 \mathrm{~dB}$ and $1.7 \mathrm{~dB}$ as compared to their equivalent systems 8QAM and 16QAM, similarly, 4-FSIM-16QAM achieves a gain of $2.2 \mathrm{~dB}$. It is worth mentioning that FSIM maintains its superiority in a frequency selective channel compared to existing SC SISO-IM schemes where the former achieves a minimum gain of 4 to $6 \mathrm{~dB}$. Thus, the proposed scheme demonstrates that ISI is not necessarily undesirable while it is controllable and predictable, since it permits to achieve a higher system capacity compared to systems that enforce zero interference. Finally, it can be concluded that FSIM scheme can achieve even higher SE after designing a larger filter bank (higher $N$ ), and it is useful for ultra-high rate wireless communication.

Consequent to the results presented in this paper, our current and future research will focus on optimal filter bank design for this scheme and its equalization to further improve the system SE and performance. In addition, the novel Filter IM domain will be extended to MIMO mode, and more schemes in the Filter IM domain are being explored.

\section{APPENDIX}

\section{PROOF OF THE EQUIVALENCY BETWEEN CONVENTIONAL PULSE-SHAPING AND THE PROPOSED SYSTEM}

The pulse-shaping in conventional transmitter is a discrete convolution of $c^{\prime \prime}[m]$ the complex symbols up-sampled by $\lambda$ with the finite impulse response (FIR) pulse-shaping filter $f[m]$ of length $L$. Thus, the proposed system becomes equivalent to conventional transceiver when all filters in the bank are the same Nyquist filter $f$. In this case, the ISI cancellation at the receiver can be deactivated. The conventional pulseshaping can be expressed as:

$$
\begin{aligned}
x[m] & =f[m] * c^{\prime \prime}[m]=\sum_{m=-\infty}^{\infty} f[m] \cdot c^{\prime \prime}[n-m] \\
& =\sum_{m=1}^{L} f[m] . c^{\prime \prime}[n-m],
\end{aligned}
$$

where $f[m]=0$ when $m$ is outside the region $[1, L], c^{\prime \prime}[m]$ is of length $N \cdot \lambda$ and $N$ is the number of APM symbols.

Using "Overlap-Add method", the output of the filtering convolution can be divided into multiple convolutions of $f[\mathrm{~m}]$ with segments of $c^{\prime \prime}[m]$ :

$$
c_{n}^{\prime \prime}[m]= \begin{cases}c^{\prime \prime}[m+n \cdot \lambda] & m=1,2, \ldots, \lambda \\ 0 & \text { otherwise }\end{cases}
$$

Then:

$$
c^{\prime \prime}[m]=\sum_{n} c_{n}^{\prime \prime}[m-n \cdot \lambda]
$$

Thus, $x[m]$ can be written as a sum of convolutions by replacing (33) in (31), we obtain:

$$
\begin{aligned}
x[m] & =f[m] * c^{\prime \prime}[m]=f[m] *\left(\sum_{n} c_{n}^{\prime \prime}[m-n . \lambda]\right) \\
& =\left(\sum_{n} f[m] * c_{n}^{\prime \prime}[m-n \cdot \lambda]\right) \\
& =\sum_{n} s_{n}[m-n . \lambda],
\end{aligned}
$$

where $s_{n}[m] \triangleq f[m] * c_{n}^{\prime \prime}[m]=f[m] * c_{n}^{\prime}[m]=$ is zero outside the region $[1, L]$ because $c_{n}^{\prime \prime}[m]$ and $c_{n}^{\prime}[m]$ contains only one non-zero element for each $n$.

In the proposed system, $s_{n}[m]$ is calculated for each APM symbol, then these outputs are overlapped and added but the filter $f$ can be changed at each symbol period to gain in SE by indexing the selected filter.

\section{ACKNOWLEDGMENT}

The research leading to these results received funding from the French National Research Agency (ANR-17-CE25-0013) within the frame of the project BRAVE.

\section{REFERENCES}

[1] R. Y. Mesleh, H. Haas, S. Sinanovic, C. W. Ahn and S. Yun, "Spatial modulation," IEEE Trans. Veh. Technol., vol. 57, no. 4, pp. 2228-2241, July 2008.

[2] A. Younis, N. Serafimovski, R. Mesleh, and H. Haas, "Generalised spatial modulation," in Proc. Signals, Syst. Comput., pp. 1498- 1502, 2010.

[3] J. T. Wang, S. Y. Jia, J. Song, "Generalised spatial modulation system with multiple active transmit antennas and low complexity detection scheme," IEEE Trans. Wireless Commun., vol.11, no. 4, pp. 1605-1615, Apr. 2012.

[4] P. Liu, M. Di Renzo and A. Springer, "Variable- $N_{u}$ generalized spatial modulation for indoor LOS mmWave communication: Performance optimization and novel switching structure," IEEE Trans. Commun., vol. 65 no. 6, pp. 2625-2640, June 2017.

[5] E. Basar, U. Aygolu, E. Panayirci, and H. V. Poor, "Orthogonal frequency division multiplexing with index modulation," IEEE Trans. Signal Process., vol. 61, no. 22, pp. 5536-5549, 2013.

[6] M. Nakao, T. Ishihara, and S. Sugiura, "Single-carrier frequency domain equalization with index modulation," IEEE Commun. Lett., vol. 21, no. 2, pp. 298-301, Feb. 2017.

[7] M. Chafii, F. Bader and J. Palicot, "SC-FDMA with index modulation for M2M and IoT uplink applications," in 2018 IEEE Wireless Commun. and Netw. Conf. (WCNC), Barcelona, 2018, pp. 1-5.

[8] E. Başar, "Multiple-input multiple-output OFDM with index modulation,' IEEE Signal Process. Lett., vol. 22, no. 12, pp. 2259-2263, Dec. 2015.

[9] S. Sugiura, S. Chen, and L. Hanzo, "Coherent and differential space-time shift keying: A dispersion matrix approach,” IEEE Trans. Commun., no. 11, pp. 3219-3230, Nov. 2010.

[10] S. Sugiura, C. Xu, S. X. Ng and L. Hanzo, "Reduced-complexity coherent versus non-coherent QAM-aided space-time shift keying," IEEE Trans. Commun., vol. 59, no. 11, pp. 3090-3101, Nov. 2011.

[11] M. I. Kadir, S. Sugiura, J. Zhang, S. Chen and L. Hanzo, "OFDMA/SCFDMA aided space-time shift keying for dispersive multiuser scenarios," IEEE Trans. Veh. Technol., vol. 62, no. 1, pp. 408-414, Jan. 2013.

[12] I. A. Hemadeh, M. El-Hajjar, S. Won and L. Hanzo, "Multi-set spacetime shift-keying with reduced detection complexity," IEEE Access, vol. 4, pp. 4234-4246, 2016.

[13] H. A. Ngo, C. Xu, S. Sugiura and L. Hanzo, "Space-time-frequency shift keying for dispersive channels," IEEE Signal Process. Lett., vol. 18, no. 3, pp. 177-180, Mar. 2011.

[14] French funded project-ANR-17-CE25-0013, "Back to single-carrier for beyond-5G communications above $90 \mathrm{GHz}-(\mathrm{BRAVE})$, ' [Online]. Available: http://www.brave-beyond5g.com/.

[15] Y. Corre, G. Gougeon, J-B Doré, S. Bicaïs, B. Miscopein, E. Faussurier, M. Saad, J. Palicot, F. Bader, "Sub-THz spectrum as enabler for 6G wireless communications up to $1 \mathrm{Tbit} / \mathrm{s}$," in 6G Wireless Summit, March 2019, Levi Lapland, Finland.

[16] M. Saad, F. Bader, J. Palicot, Y. Corre, G. Gougeon, J-B Doré, "Beyond$5 \mathrm{G}$ wireless Tbps scenarios and requirements," French funded projectANR-17-CE25-0013 BRAVE, Tech. Report BRAVE D1.0, 2018. [Online]. Available: https://hal.archives-ouvertes.fr/hal-01947363/document.

[17] M. Saad, F. Bader, J. Palicot, A. C. Al Ghouwayel, H. Hijazi, "Single carrier with index modulation for low power terabit systems," in the Proc. of the IEEE Wireless Commun. and Netw. Conf. (WCNC), Marrakech, Morocco. Apr. 2019.

[18] M. Saad, F. Lteif, A. C. Al Ghouwayel, H. Hijazi, J. Palicot and F. Bader, "Generalized spatial modulation in highly correlated channel," IEEE International Symposium on Personal Indoor and Mobile Radio Communications (PIMRC), Istanbul, Turkey, Sept. 2019. 
[19] M. Saad, F. Bader, A. C. Al Ghouwayel, H. Hijazi, N. Bouhel and J. Palicot, "Generalized Spatial Modulation for Wireless Terabits Systems Under Sub-THZ Channel With RF Impairments," ICASSP 2020 - 2020 IEEE International Conf. on Acoustics, Speech and Signal Processing (ICASSP), Barcelona, Spain, 2020, pp. 5135-5139.

[20] M. Saad, A. C. Al Ghouwayel, H. Hijazi, F. Bader and J. Palicot,"MIMO Techniques for Wireless Terabits Systems under sub-THz Channel with RF Impairments," IEEE International Conf. on Commun. (ICC), Dublin, Ireland, June 2020.

[21] J. Pierce, "Optical channels: Practical limits with photon counting," IEEE Trans. Commun., vol. COM-26, no. 12, pp. 1819-1821, Dec. 1978.

[22] X. Liu, T. H. Wood, R. W. Tkach and S. Chandrasekhar, "Demonstration of record sensitivity in an optically pre-amplified receiver by combining PDM-QPSK and 16-PPM with pilot-assisted digital coherent detection," Optical Fiber Commun. Conf. and Exposition and the National Fiber Optic Engineers Conf., Los Angeles, CA, 2011, pp. 1-3.

[23] S. Althunibat, R. Mesleh and T. F. Rahman, "A novel uplink multiple access technique based on index-modulation concept," IEEE Trans. Commun., vol. 67, no. 7, pp. 4848-4855, July 2019.

[24] S. Althunibat, R. Mesleh and K. Qaraqe, "Quadrature Index Modulation Based Multiple Access Scheme for 5G and Beyond," in IEEE Commun. Lett., vol. 23, no. 12, pp. 2257-2261, Dec. 2019.

[25] S. Althunibat, R. Mesleh and K. A. Qaraqe, "IM-OFDMA: A Novel Spectral Efficient Uplink Multiple Access Based on Index Modulation," in IEEE Trans. Veh. Technol,, vol. 68, no. 10, pp. 10315-10319, Oct. 2019.

[26] D. Tsonev, S. Sinanovic, and H. Haas, "Enhanced subcarrier index modulation (SIM) OFDM," in Proc. IEEE GLOBECOM Workshops, Dec. 2011, pp. 728-732

[27] E. Öztürk, E. Basar and H. A. Çırpan, "Generalized frequency division multiplexing with flexible index modulation numerology," IEEE Signal Process. Lett., vol. 25, no. 10, pp. 1480-1484, Oct. 2018.

[28] R. Fan, Y. J. Yu and Y. L. Guan, "Generalization of orthogonal frequency division multiplexing with index modulation," IEEE Trans. Wireless Commun., vol. 14, no. 10, pp. 5350-5359, Oct. 2015.

[29] M. Wen, B. Ye, E. Basar, Q. Li and F. Ji, "Enhanced orthogonal frequency division multiplexing with index modulation," IEEE Trans. Wireless Commun., vol. 16, no. 7, pp. 4786-4801, July 2017.

[30] G. Cheng, L. Wang, W. Xu and G. Chen, "Carrier index differential chaos shift keying modulation," in IEEE Trans. Circuits and Systems II: Express Briefs, vol. 64, no. 8, pp. 907-911, Aug. 2017.

[31] G. Kaddoum, Y. Nijsure and H. Tran, "Generalized code index modulation technique for high-data-rate communication systems," in IEEE Trans. Veh. Technol., vol. 65, no. 9, pp. 7000-7009, Sept. 2016.

[32] A. M. Jaradat, J. M. Hamamreh and H. Arslan, "OFDM with subcarrier number modulation," IEEE Wireless Commun. Lett., vol. 7, no. 6, pp. 914-917, Dec. 2018.

[33] S. Dang, G. Ma, B. Shihada and M. Alouini, "Enhanced orthogonal frequency-division multiplexing with subcarrier number modulation," IEEE Internet of Things Journal, vol. 6, no. 5, pp. 7907-7920, Oct. 2019.

[34] T. Ishihara and S. Sugiura, "Faster-than-nyquist signaling with index modulation," IEEE Wireless Commun. Lett., vol. 6, no. 5, pp. 630-633, Oct. 2017.

[35] M. Nakao and S. Sugiura, "Spectrally efficient frequency division multiplexing with index-modulated non-orthogonal subcarriers," IEEE Wireless Commun. Lett., vol. 8, no. 1, pp. 233-236, Feb. 2019.

[36] M. Nakao, T. Ishihara and S. Sugiura, "Dual-mode time-domain index modulation for nyquist-criterion and faster-than-nyquist single-carrier transmissions," IEEE Access, vol. 5, pp. 27659-27667, 2017.

[37] M. Nakao and S. Sugiura, "Dual-mode time-domain single-carrier index modulation with frequency-domain equalization," in 2017 IEEE 86th Veh. Technol. Conf. (VTC-Fall), Toronto, Ontario, 2017, pp. 1-5.

[38] T. Mao, Z. Wang, Q. Wang, S. Chen and L. Hanzo, "Dual-mode index modulation aided OFDM," IEEE Access, vol. 5, pp. 50-60, 2017.

[39] T. Mao, Q. Wang and Z. Wang, "Generalized dual-mode index modulation aided OFDM," IEEE Commun. Lett., vol. 21, no. 4, pp. 761-764, Apr. 2017.

[40] M. Wen, E. Basar, Q. Li, B. Zheng and M. Zhang, "Multiple-Mode Orthogonal Frequency Division Multiplexing With Index Modulation," in IEEE Trans. Commun., vol. 65, no. 9, pp. 3892-3906, Sept. 2017.

[41] M. Wen, Q. Li, E. Basar and W. Zhang, "Generalized Multiple-Mode OFDM With Index Modulation," in IEEE Trans. Wireless Commun., vol. 17, no. 10, pp. 6531-6543, Oct. 2018.

[42] J. Li, S. Dang, M. Wen, X. Jiang, Y. Peng and H. Hai, "Layered Orthogonal Frequency Division Multiplexing With Index Modulation," in IEEE Systems Journal, vol. 13, no. 4, pp. 3793-3802, Dec. 2019.
[43] T. Mao, Q. Wang, Z. Wang and S. Chen, "Novel Index Modulation Techniques: A Survey," in IEEE Commun. Surveys \& Tutorials, vol. 21, no. 1, pp. 315-348, Firstquarter 2019.

[44] F. Pancaldi, G. M. Vitetta, R. Kalbasi, N. Al-Dhahir, M. Uysal and H. Mheidat, "Single-carrier frequency domain equalization," in IEEE Signal Process. Mag., vol. 25, no. 5, pp. 37-56, September 2008.

[45] K. Berberidis and P. Karaivazoglou, "A block adaptive DFE in the frequency domain based on tentative decisions," in 9th Eur. Signal Process. Conf. (EUSIPCO 1998), Rhodes, 1998, pp. 1-4.

[46] J. Proakis and M. Salehi, Digital communications. Boston, MA: McGraw-Hill, 2008.

[47] E. Seifi, M. Atamanesh, and A. K. Khandani, "Media-based MIMO: A new frontier in wireless communication," Oct. 2015. [Online]. Available: arxiv.org/abs/1507.07516

[48] A. Mokh, M. Helard and M. Crussiere, "Space Shift Keying Modulations for Low Complexity Internet-of-Things Devices," GLOBECOM 2017 . IEEE Global Commun. Conf., Singapore, 2017, pp. 1-7.

[49] A. K. Khandani, "Media-based modulation: A new approach to wireless transmission," 2013 IEEE International Symposium on Information Theory, Istanbul, 2013, pp. 3050-3054.

[50] E. Basar, "Media-Based Modulation for Future Wireless Systems: A Tutorial," in IEEE Wireless Communications, vol. 26, no. 5, pp. 160-166, October 2019.

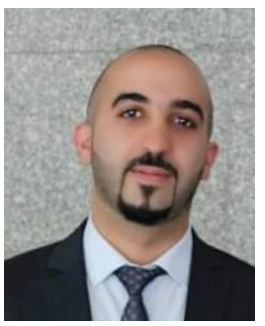

Majed Saad received the M. Sc degree with the highest distinction in computer and communication engineering (CCE) in 2016 from Lebanese International University (LIU), Beirut, Lebanon. He secured the first rank during his M.Sc. studies in the CCE Department, all LIU campuses, Lebanon. From October 2016, he was a Lab Instructor at the school of engineering of LIU. He is currently pursuing a Ph.D. in signal processing and communication systems at CentraleSupélec, France. His general research interests include beyond 5G, millimeter, sub- $\mathrm{THz}$ and Terahertz systems, MIMO systems, Index Modulation, signal processing, and equalization. Since January 2018, he is involved in the BRAVE French national project (ANR) about Beyond 5G Terabits wireless communication in the sub-TeraHertz bands. He received a grant from CNRS GdR-ISIS in France for mobility and cooperation with the University of Patras, Greece, in 2019. He also served as a Reviewer for several IEEE conferences and IEEE Transactions on Communications.

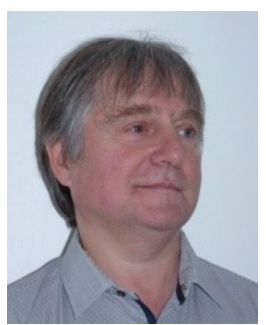

Jacques Palicot received, in 1983, his Ph.D. degree in signal processing from the University of Rennes. $\mathrm{He}$ is now Emeritus Professor with CentraleSupelec, formerly Supélec. He was head of the Signal, Communications and Embedded Electronics (SCEE) research team until January 2019. His research focuses on adaptive signal processing for digital communications, automatic measurements techniques, software radio, cognitive radio, and Green Radio. He has taken an active part in various international bodies EBU, CCIR, URSI, and within RACE, ACTS and IST European projects $\mathrm{He}$ is author or co-author of more than 380 papers among them more than 80 internationals journals, three books and 25 patents. He served as Associate Editor for EURASIP JASP (2008-19). He also served as lead guest editor for Special Issues on Software Radio, Cognitive Radio and Green Radio. He has been General Chairman of ICT 2018, Next-GWiN 2014, ISCIT 2011. Technical Program Chairman of CROWNCOM 2009,2016 GREENCOM 2013, CRN Symposium ICC 2014. He is Fellow member of SEE since 2016 and he received the URSI-France medal in 2020. 


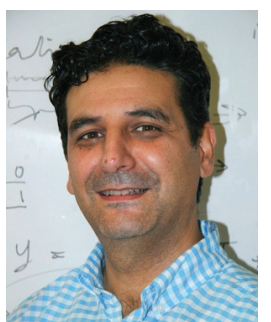

Faouzi Bader (M'02-SM'07) received the Ph.D. degree (Hons.) in telecommunications from the Universidad Politécnica de Madrid, Madrid, Spain, in 2002. He joined the Centre Technològic de Telecomunicacion de Catalunya-CTTC, Barcelona-Spain, as Research Associate in 2002, and he has been Senior Research Associate from 2006 to 2013. Since June 2013-2019, he has been Associate Professor at CentraleSupélec, France. Since 2017, he is Honorary Adjunct Professor with University Technology Sydney-UTS, Australia. Starting 2020, he is the Director of Research at the "Institut Supérieur d'Électronique de Paris"ISEP, France. His research activities are mainly on ICT advanced systems as: $\mathrm{THz}$ wireless communications, $5 \mathrm{G}$ and beyond networks, and cognitive radio communication environments. He has been involved in several European projects from the 5th-7th EC research frameworks, and the general coordinator and manager of the EC funded ICT "EMPhAtiC" project focusing on "Enhanced Multicarrier Techniques for Professional Ad-Hoc and Cell-Based Communications". He has published over 34 journals and 132 papers in peerreviewed international conferences, 13 book chapters, and 5 edited books.

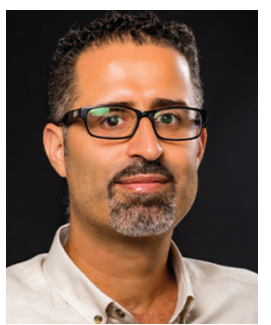

Ali Chamas Al Ghouwayel received his B.E. degree in Physics-Electronics in 2002 from the Lebanese University, Beirut, his B.E. degree in Electronics Engineering and his M.S. Degree in Telecommunications in 2004 from the National School of Engineering of Brest (ENIB), Brest, France, and his Ph.D. in Signal Processing and Telecommunications in 2008 from the High School of Electricity (Supélec) and University of Rennes 1, Rennes, France. His research activities concerned Parametrization Study for Software Defined Radio Systems. In 2008, He joined the Lab-STICC laboratory in Lorient, France and worked as a PostDoctoral researcher on the European Project DAVINCI. In 2010, He joined the Lebanese International University, in Beirut, Lebanon, as Assistant Professor and then promoted to the rank of Associate Professor in 2016. His current research interests include Study, Optimization, and Adequation AlgorithmArchitecture for Hardware Implementation of ultra-throughput Non-Binary LDPC decoders on FPGA and ASIC, Internet of Things (IoT) Frame detection and synchronization at very low SNR. He participated as Principal Investigator in the ANR Project (QCSP: Quasi-Cyclic Short Packet) involving a consortium of academic and industrial partners (Orange Labs, SEQUANS, CEA, Lab-STICC UBS, IMT-Atlantic, ....). The aim of the QCSP project, is to contribute to the design of future Internet of Things (IoT) networks by defining, implementing, and testing a new coded modulation scheme dedicated to IoT networks. He has several refereed journal and conference papers. He also served as Reviewer for several IEEE Transactions Journals and TPC for IEEE Conferences. In August 2020, he joined the School of Engineering EFREI Paris as Chair of the Major Avionics and Space.

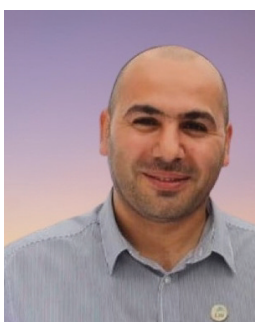

Hussein Hijazi received his B.E. degree in Computer and Communications Engineering in 2004 from the Lebanese University, Beirut, Lebanon, his M.S. degree and Ph.D. in Signal Processing and Communications in 2005 and 2008 respectively from Polytechnic Institute of Grenoble (Grenoble-INP), Grenoble, France. Dr. Hijazi earned a Ph.D. Award 2010 at Polytechnic Institute of Grenoble, Grenoble, France. From September 2008 to August 2009, he was an assistant professor at the schools of engineering ENSIMAG, PHELMA and ENSE3 of GrenobleINP. From September 2009 to December 2009, he was a Research engineer in telecommunications at National Center for Scientific Research (CNRS) University of Lille 1, TELICE Laboratory. From December 2009 to December 2010, he was a Post-doctoral researcher at France Telecom-orange Labs, RD center, Meylan, France. Since 2010, he has joined the computer and communications engineering department at Lebanese International University (LIU) where he is currently an Associate Professor. His general research interests lie in the areas of signal processing and communications, including synchronization, time-varying channel estimation and equalization algorithms for wireless digital communications, multi-carrier and MIMO systems, modulation index, beyond-5 $\mathrm{G}$ communications above $90 \mathrm{GHz}$ (Terabit systems) and IoT technologies: detection and synchronization. 